\title{
MicroRNA Member TaMIR5062 in T. Aestivum Involves Plant Drought and Salt Acclimation via Regulating Physiological Processes Associated with Water Retention and ROS Homeostasis
}

\author{
Yuanjinzi Qiao \\ Hebei Agricultural University \\ Ling Wang \\ Hebei Agricultural University \\ Zidi Yu \\ Hebei Agricultural University \\ Chenyang Ni \\ Hebei Agricultural University \\ Tianjiao Li \\ Hebei Agricultural University \\ Chengjin Guo \\ Hebei Agricultural University \\ Kai Xiao ( $\nabla$ xiaokai@hebau.edu.cn ) \\ Hebei University of Architecture
}

\section{Research Article}

Keywords: wheat (Triticum astivum L.), P deprivation, miRNA member, drought and salt stresses, plant growth, physiological traits, antioxidant enzymes

Posted Date: December 16th, 2021

DOI: https://doi.org/10.21203/rs.3.rs-1137207/v1

License: (c) (i) This work is licensed under a Creative Commons Attribution 4.0 International License. Read Full License 


\section{Abstract}

microRNA members negatively regulate target genes via posttranscriptional cleavage or translation repression mechanisms, impacting on plant growth, development, and stress response. In this study, we characterized TaMIR5062, a miRNA member in T. aestivum, in mediating drought and salt responses. TaMIR5062 interacts with six target genes, including two encoding calmodulins, three coding for 4oxalocrotonate tautomerases, and one for pumilio-family RNA binding domain protein. The TaMIR5062 transcripts were gradually upregulated in plants upon 27-h drought and salt treatments, whose induced expression under stress treatments was restored following the normal recovery condition. Tobacco $(N$. tabacum) lines transformed with TaMIR5062 modified growth traits under drought and salt treatments; the lines overexpressing miRNA (i.e., Sen 1 and Sen 2) improved growth traits (i.e., biomass, leaf area, and root length) whereas those with knockdown (Anti 1) alleviated growth features compared with wild type. These results suggested the critical role of TaMIR5062 in improving plant drought and salt tolerance. In line with growth traits in stress-challenged lines, improved leaf water retention (i.e., promoted stomata closing, water losing rate, and osmolytes) and ROS-associated parameters (higher SOD, CAT, and POD activities, etc.) were shown in Sen 1 and Sen 2 under stress conditions. Antioxidant enzyme (AE) genes NtMnSOD1, NtCAT, and NtPOD9 encoding SOD, CAT, and POD, respectively, enhanced transcription in Sen 1 and downregulated expression in Anti 1 challenged with drought and salt stress. These results suggested the improved ROS homeostasis mediated by TaMIR5062 associates modified expression of distinct AE genes. Quantities of genes functional into categories "biological process", "cellular component", and "molecular function" contribute to TaMIR5062-mediated osmotic stress adaptation by regulating distinct biological pathways (i.e., protein folding) and metabolisms (i.e., photosynthesis and isoprenoid biosynthesis), which impact on plant osmotic-regulation, ROS homeostasis, and stress defensiveness underlying miRNA regulation. TaMIR5062 is a valuable target for molecular breeding of drought-tolerant crop cultivars.

\section{Key Message}

TaMIR5062 confers plants drought and salt stress adaptation via modulation of physiological processes associated with stomata movement, osmolyte biosynthesis, and ROS homeostasis.

\section{Introduction}

As the frequently occurred stresses to plants, drought and high salinity alleviate drastically cell function, negatively regulating plant growth, development, and the yield formation capacity for cereal crops (Cattivelli et al. 2008, Maiti et al. 2014). Under osmotic stress conditions (i.e., drought and salt stress), a large set of physiological processes associated with plant growth behavior including root system architecture (RSA) establishment, protein biosynthesis metabolism, photo-chemical efficiency and assimilate production in photosystem, biosynthesis of osmolytes, and differentiation and establishment of tissues are altered, which act with synergistic manner in regulating the growth traits and yield formation potential of crop plants (Benjamin and Nielsen 2006; Zhao et al. 2019; Naheed et al. 2021). 
Elite cultivars sharing strong capacities to cope with drought and salt stresses have been potential in improving productivity of the crop plants cultivated under water-saving and high salinity conditions.

Plants have evolved a suite of strategies to acclimate to adverse osmotic stress conditions through various mechanisms initiated at physiological and molecular levels (Chaves et al. 2009; Fang and Xiong 2015; Yang and Gao 2018). Thus far, the knowledge as to the molecular networks mediated by drought and salt signaling has been extensively developed, given the advanced research approach established in past several decades. A quantity of critical regulators functional in plant response to osmotic stressors has been identified, which has benefited the elucidation of biochemical pathways and action mechanisms underlying drought and salt stress stressors. Among them, signaling receptors, kinases in the families of mitogen-activated protein kinases (MAPK) and calcium-dependent protein kinases (CDPK), and transcriptional regulators (i.e. calmodulin-binding proteins, phosphatases, and distinct transcription factors), act in a cooperate mode in defining plant stress response, given their crucial roles in mediating transduction of the stress signaling (Danquah et al. 2014; Delormel and Boudsocq 2019; Yoshida et al. 2014). Therefore, plant responses to osmotic stresses are determined largely by the signaling transduction system. Functional characterization on the critical regulators constituting the system can help depth dissection of the molecular mechanisms underlying plant osmotic stress responses.

Members of the microRNA (miRNA) family are non-coding RNA molecules with nucleotide acids from 20 to $24 \mathrm{nt}$ in length. They are involved in the modulation of quantities of physiological processes through regulating target genes at posttranscriptional or translational level with a negative regulation mechnasim (Ferdous et al. 2015; Sun et al. 2019). Thus far, characterization on miRNA members in plant species has validated their function as critical mediators in diverse biological pathways, being associated with growth and development, such as growth phase transition, leaf morphogenesis, floral organ identity, and root architecture establishment (Lu and Huang 2008; Rubio-Somoza and Weigel 2011). Additionally, a large set of miRNA members also act as crucial modulators in plant responses to adverse abiotic stresses, through regulating transcription of the stress defensiveness-associated genes (Phillips et al. 2007; Lu and Huang 2008; Mazzucotelli et al. 2008; Shukla et al. 2008; Lewis et al. 2009). For instance, member miR319 in Arabidopsis confers plant responses to a set of stress conditions, including water deficit, high salinity, and extreme low temperature, which is largely ascribed to its modified transcription efficiency upon these stressors (Sunkar and Zhu 2004; Liu et al. 2008; Lv et al. 2010; Thiebaut et al. 2012; Zhou et al. 2010; Kar and Raichaudhuri 2021). Altered expression of this miRNA leads to significant variation on transcripts of genes in the TCP transcription factor (TF) family encoding basic helix-loop-helix (bHLH) proteins, endowing plants increased adaptation to above stress conditions (Palatnik et al. 2003; Ori et al. 2007; Nag et al. 2009). In cereal crops, a subset of miRNA members is responses to drought stress, contributing to improved adaptation of plants to this stressor (Zhou et al. 2010; Qiu et al. 2020). Likewise, the miRNA members involving plant salt stress have also been documented extensively in various plant species to date, including miR393, miR319c, miR389a.1 in A. thaliana (Sunkar and Zhu 2004), miR169, miR319, miR393, miR396c, miR398, miR528, miR820, miR1848 in O. sativa (Haldar and Bandyopadhyay 2021), mi408 in T. aestivum (Bai et al. 2018), miR156j, miR156k囚miR159a, miR159b, miR162a, miR162b, miR167a, miR168a, miR169g, miR169f, etc. in Z. mays (Ding et al. 2009; Zhao et al. 2009), and miR156d, 
miR164a, miR393a, miR319a and miR172b in barley (Lv et al. 2012). However, although the miRNA members and characterization on them were extensively investigated, the mechanisms underlying miRNA-mediated responses to osmotic stresses (i.e., drought and salt stress) are needed to be further characterized in plant species, especially in cereal ones.

Wheat ( $T$. aestivum) acts as one of the important cereal crops and is extensively cultivated around the world, contributing greatly to the food supply security worldwide. However, the cultivation process of wheat is frequently challenged by the shortage of water resource, leading to drought stress for the crop plants. Additionally, large area for wheat planting is also grown in the environment with high salt contents, resulting in the alleviated productivity of crops due to high $\mathrm{Na}^{+}$toxicity (Terletskaya et al. 2013). Therefore, improvement of plant adaptation to drought and salt stress can benefit promotion of the sustainably agricultural development in developing countries. Thus far, although the miRNA database in T. aestivum (www.mirbase.org, release 21) deposits a large set of members of the miRNA family and a suite of investigations have reported the characterization of miRNAs, target genes, and putative miRNA/target interaction behaviors (Sun et al. 2014; Wang et al. 2014; Bakhshi et al. 2017), the mechanisms as to how distinct miRNA members of wheat mediate plant osmotic stress responses are largely to be determined. In this study, we characterized TaMIR5062, a miRNA member in T. aestivum, in mediating plant adaptation to drought and salt stresses. Our results suggested that TaMIR5062 functions a crucial mediator in regulating above stressors through regulating the physiological processes associated with osmotic stress response.

\section{Materials And Methods}

\section{Characterization of TaMIR5062}

Our previous investigation with an aim to identify wheat miRNA family members in response drought stress revealed that TaMIR5062 (accession number MI0030391) was upregulated on transcripts in plants (cv. Shimai 22) challenged with this stressor. This finding prompted us to further characterize it in mediating plant osmotic stress response in more detail. Sequences of TaMIR5062 at precursor and mature conditions and structure of stem-loop feature initiated by the miRNA precursor were derived from the miRNA information deposited in database of T. aestivum (www.mirbase.org).

\section{Prediction of the target genes of TaMIR5062}

An online tool psRNATarget (Plant microRNA Potential Target Finder; http://plantgrn.noble.org/psRNATarget/) was used to predict the target genes interacted by TaMIR5062 using the default parameters suggested. The target genes were identified by search against the CDNA deposits referred to as cDNA databases of $T$. aestivum (bread wheat), cDNA, EnsemblPlant, release 43. Putative biological roles of the target genes were determined through BLASTn search analyses supplemented in National Center for Biotechnology Information (NCBI) (www. ncbi.nlm.nih.gov/), with cDNA sequences of target genes used as queries 


\section{Expression analysis of TaMIR5062}

The seedlings of wheat (cv. Shimai 22) cultivated under different conditions, including normal growth, drought, and salt stress were used to determine the expression patterns of TaMIR5062. In brief, the seedlings after germination were firstly cultured in a standard Murashige and Skoog (MS) solution to the third leaf stage under following growth condition: a photoperiod range of $12 \mathrm{~h} / 12 \mathrm{~h}$ (day/night) with light intensity of $300 \mu \mathrm{mol} \mathrm{E} \mathrm{m}{ }^{-2} \mathrm{~s}^{-1}$ during light phase, temperature range of $28^{\circ} \mathrm{C} / 23^{\circ} \mathrm{C}$ (day/night), and relative humidity from 65 to $70 \%$. At that time, they were subjected to treatments of simulated drought and salt stress. Of which, the drought treatment was established by growing an aliquot of seedlings in a modified MS solution supplemented with PEG-6000 (10\%, w/v) whereas salt treatment was initiated by culturing seedlings in a MS solution containing $200 \mathrm{mM} \mathrm{NaCl}$. Tissues of leaf and root were sampled at time points of $0 \mathrm{~h}$ (prior to treatment), and $3 \mathrm{~h}, 6 \mathrm{~h}, 9 \mathrm{~h}, 12 \mathrm{~h}$, and $27 \mathrm{~h}$ after the treatments. In addition,

aliquots of the seedlings after $27 \mathrm{~h}$ of drought and salt treatments were re-subjected to the normal growth condition to understand regular recovery response of TaMIR5062. The normal recovery treatments were established by transferring the drought- and salt-stressed seedlings ( $27 \mathrm{~h}$ after treatments) into standard MS solution. Likewise, tissues mentioned were sampled at time points of $3 \mathrm{~h}, 6 \mathrm{~h}, 9 \mathrm{~h}, 12 \mathrm{~h}$, and $27 \mathrm{~h}$ following recovery treatments. qRT-PCR was performed to evaluate the transcripts of TaMIR5062 in samples collected as previously described (Guo et al. 2013). With this purpose, total RNA in samples was extracted using TRIzol reagent (Invitrogen, USA). The first-strand cDNA was synthesized using RT-AMV transcriptase (TaKaRa, Dalian, China) from total RNA $(2 \mu \mathrm{g})$ after removal of putative genomic DNA treated with RNase-free DNase (TaKaRA, Dalian, China). qRT-PCR was conducted in a volume of $25 \mu \mathrm{L}$ which contained following components: $12.5 \mu \mathrm{L}$ of SYBR Premix ExTaqTM (TaKaRa, Dalian, China), 0.5 $\mu \mathrm{L}$ each for forward and reverse primers, $1 \mu \mathrm{L} \mathrm{cDNA}$ and $10.5 \mu \mathrm{L}$ nuclease-free water. The transcripts of TaMIR5086 were calculated according to the $2^{-\triangle \Delta C T}$ formula, using a constitutive gene Tatubulin in $T$. aestivum as an internal standard to normalize the target expression levels. Specific primers used for amplification of TaMIR5062 and Tatubulin are shown in Table S1.

\section{Assays of the growth traits in transgenic lines}

Tobacco (N. tabacum) lines with overexpression or knockdown expression of TaMIR5062 were established to characterize the role of this miRNA in mediating drought and salt responses. With this purpose, we performed RT-PCR to amplify the precursor of TaMIR5062 using specific primer pair (Table S1), then inserted it into the restriction sites $\mathrm{Ncol} / \mathrm{BstEl}$ in binary vector pCAMBIA3301 under the control of the CaMV35Spromoter. The expression cassettes harboring TaMIR5062 in sense and antisense orientations were separately integrated into A. tumefaciens strain EHA105 using heat-shock approach and subjected to genetic transformation onto N. tabacum (cv. Wisconsin 35) as described previously (Shi et al. 2020). Transcripts of the target miRNA in transgenic lines were evaluated based on qRT-PCR performed to be similar for evaluating expression levels of TaMIR5062 upon stress conditions.

Three lines at T3 generation, including two with more TaMIR5062 transcripts (Sen 1 and Sen 2) and one less transcript of TaMIR5062 homolog (Fig. S1), were selected to be subjected to various growth 
treatments, including normal growth condition, drought stress, and salt treatment. Of which, the normal growth condition was established by growing plants of the transgenic lines (i.e., Sen 1, Sen 2, and Anti 1) together with wild type (control without transformed with TaMIR5062, WT) in the standard MS solution; drought treatment was initiated by culturing the transgenic and WT plants in a modified MS solution supplemented with PEG-6000 (5\%, w/v); salt treatment was established by culturing transgenic and WT plants in MS solution containing $150 \mathrm{mM} \mathrm{NaCl}$. After five weeks of treatments, phenotypes, biomass in aerial and root tissues, leaf area, root lengths and root fresh weights were assessed. Of which, phenotypes were recorded as images taken by a digital camera; biomass in aerial tissues, roots, and plants were obtained from the representative samples after oven-drying; leaf area, root length, and root fresh weights in plants were determined following conventional approach.

\section{Assays of stomata stomatal behavior and leaf water retention capacity in transgenic lines}

The stomata closing feature and water retention capacity of leaf that impact largely on plant response to osmotic stresses were assessed in the transgenic lines (Sen 1, Sen 2, and Anti 1) and WT under drought and salt stress conditions. To characterize stomata movement, the plants of Sen 1, Anti 1, and WT grown under normal condition were subjected to drought and salt treatments mentioned above. At $0 \mathrm{~h}$ (prior to treatment), and $0.25 \mathrm{~h}, 0.5 \mathrm{~h}$, and $1 \mathrm{~h}$ following stress conditions, leaf samples in transgenic and WT plants were collected. Stomata nature in sampled leaves was observed under microscope after fixation using nail polish oil as described previously (Ding et al. 2014). At least fifty of representative stomata were observed in each sample. Stomata aperture properties were observed under a microscope at the indicated times. Water retention capacities were analyzed using detached representative leaves of transgenic (Sen 1 and Anti 1) and WT plants collected after $0 \mathrm{~h}, 2 \mathrm{~h}, 4 \mathrm{~h}$, and $6 \mathrm{~h}$ during drought and salt treatments. Fresh weights of the transgenic and WT plants under various conditions were obtained using an electronic balancer. Water losing rates (WLR) of leaves were calculated based on decreases of fresh weights at indicated times with respect to that at $0 \mathrm{~h}$.

\section{Assays of osmolyte contents in transgenic lines}

To evaluate the osmo-regulatory function mediated by TaMIR5062, the contents of soluble sugar and soluble protein were assessed in transgenic lines (Sen 1, Sen 2, and Anti 1) and WT under stress treatments, using the leaves of transgenic and WT plants treated with drought and salt as samples. The soluble sugar contents were analyzed as suggested by Hu et al. (2016) whereas the soluble protein contents were evaluated according to the approach described previously (Read and Northcote 1981).

\section{Assessments of reactive oxygen species (ROS)-associated parameters and AE gene expression in transgenic lines}

A set of reactive oxygen species (ROS)-associated parameters, including activities of antioxidant enzyme (AE, i.e., superoxide dismutase (SOD), catalase (CAT), and peroxidase (POD), contents of MDA, and membrane electric conductance (MEC), were assessed in the transgenic and WT plants after normal growth and stress treatments as aforementioned. Of which, the AE activities and MDA contents were 
analyzed as described previously (Huang et al. 2010). The MEC values in samples were determined using an electric conductance analyzer (DDS-307A, Shanghai, China) following the manufacturer' suggestion. To understand the molecular processes as to the TaMIR5062-mediated AE activities with expression patterns of AE family genes, six genes in SOD family, six genes in CAT family, and eleven genes in POD family in N. tabacum identified in NCBI GenBank database were subjected to evaluation of expression levels under various growth conditions. To this end, the transcripts of these AE genes were assessed using the transgenic and WT plants grown under normal condition, drought, and salt stress based on qRTPCR analysis, which was performed to be similar for characterization of TaMIR5062 expression mentioned previously. The genes and the gene specific primers used in qRT-PCR are shown in Table S1. Nttubulin was used as internal standard to normalize the target transcripts.

\section{RNA-seq analysis for drought-challenged transgenic lines}

The transcriptome profile upon drought underlying modulation of TaMIR5062 was investigated based on high-throughput RNA-seq analysis. With this purpose, Sen 1 and WT were cultured regularly in a standard MS solution as aforementioned. The plants of them at the fifth leaf stage were subjected to drought treatment by culturing in a MS solution supplemented with PEG-6000 $(\mathrm{w} / \mathrm{v}, 10 \%)$ for three days. After drought treatment, total RNA in samples was extracted using TRIzol reagent (Invitrogen). RNAseq libraries were constructed for the drought-challenged Sen 1 and WT plants following the procedure as described previously (Zhong et al. 2011) and subjected to generation of transcripts using Illumina HiSeq 2500 system. Putative valuable transcripts in the libraries were obtained after removal of adaptors in the reads, the transcripts with cDNA length less than $40 \mathrm{bp}$, and those being low quality based on software referred to as Trimmomatic (Bolger et al. 2014). Alignment analysis using the generated transcripts was performed by searching against the cDNA database of the reference genome $(N$. tabacum, Novogene Co, LTd, Beijing). The genes were defined differentially expressed (DE) when they exhibited 2-fold variation on transcripts between transgenic and WT plants (Robinson et al. 2010), using a false discovery rate (FDR) less than 0.05 (Benjamini and Hochberg 1995). GO terms and biochemical pathways of the DE genes were categorized using the online tool referred to as Plant MetGenMap (http://bioinfo.bti.cornell.edu/cgi-bin/MetGenMAP/home.cgi), using a CPAN pearl module during which applied as described previously (Boyle et al. 2004)

\section{Statistical analysis}

Averages of gene expression levels in qRT-PCR analysis, growth traits such as leaf area, biomass in aerial and root tissues, root fresh weights and root lengths, stomata closing rates, AE activities, MDA contents, osmolyte contents, MEC, and RNA-seq analysis in transgenic lines and WT were derived from results of four replicates. Standard errors of averages and significant differences were analyzed using the Statistical Analysis System software (SAS Corporation, Cory, NC, USA).

\section{Results}


In total of six genes are suggested to be the targets of TaMIR5062, with regulation mechanism of them underlying miRNA at posttranscriptional level. Of which, two encode calmodulin proteins, three code for 4oxalocrotonate tautomerases, and one encode pumilio-family RNA binding domain protein (Fig. 1). That half of target genes involving signaling transduction (those encoding calmodulin) and transcriptional regulation (the gene encoding RNA binding domain protein) suggests that TaMIR5062 is involved in the modulation of distinct biological processes by regulating the target genes through a cleavage mechanism.

\section{Expression patterns of TaMIR5062 under drought and salt stress conditions}

The transcripts of TaMIR5062 in aerial and root tissues were modified dramatically upon both drought and salt stresses, displaying patterns to be gradually upregulated over a $27 \mathrm{~h}$-regime of stress treatments (Fig. 2). Additionally, the stress-regulated expression of TaMIR5062 under both drought and salt treatments was restored following progression of normal recovery condition (Fig. 2). These results suggested that TaMIR5062 sensitively responds to drought and salt stress signaling in plants. That TaMIR5062 displays modified expression patterns upon stressors mentioned suggests its putative involvement in regulating plant responses to drought and salt stresses by impacting on modifying the transcripts of target genes.

\section{Growth behaviors of the TaMIR5062 transgenic lines under drought and salt treatments}

The growth traits of two lines with strong expression of TaMIR5062 (i.e., Sen 1 and Sen 2) and one with knockdown expression of target miRNA (i.e., Anti 1) (Fig. S1) were assessed under drought and salt treatments. Under normal growth condition, similar phenotypes, biomass in aerial tissue and roots, plant biomass, leaf areas, root fresh weights, and root lengths were observed among the transgenic lines and WT (Figs. 3a-3g). In contrast, under treatments of drought and salt, the transgenic lines modified the growth traits mentioned, with those shown to be drastically improved on phenotype (Fig. 3a), biomass of aerial tissue and root, and plant (Figs. 3b to 3d), leaf area (Fig. 3E), root fresh weight (Fig. 3f) and root length (Fig. 3g) in Sen 1 and Sen 2 whereas significantly alleviated on growth traits mentioned in Anti 1 compared to WT plants (Figs. 3a-3g). These results suggested that TaMIR5062 plays critical roles in mediating plant tolerance to drought and slat stresses.

\section{Stomata movement feature and water retention capacity of transgenic lines}

The stomata movement property mediated by TaMIR5062 was analyzed by assessing stomata aperture property in Sen 1 and Anti 1 treated with $1 \mathrm{~h}$ regime of drought and salt stresses. Upon these stressors, the stomata closing rates (SCR) in detached leaves were all lowered in the transgenic and WT plants; however, the decrease on closing speeds was much faster in Sen 1 and lower in Anti 1 than WT (Figs. 4a and 4b). In lines with behavior of leaf SCR shown in transgenic and WT plants, the leaf water losing rates (WSR) were in contrast to SCR values among the lines with modified TaMIR5062 expression and WT. Sen 1 showed lower whereas Anti 1 higher WSR values following progression of $6 \mathrm{~h}$ drought and salt treatments than WT plants (Figs. 4c and 4d). These results suggested that the TaMIR5086-mediated 
plant adaptation to osmotic stress is associated closely with its role in regulating water retention capacity through modulating stomata movement that affects leaf WSR behavior.

\section{Contents of soluble sugar and protein in transgenic lines under drought and salt treatments}

The contents of osmolyte (i.e., soluble sugar) and soluble protein were evaluated in stress-treated transgenic lines to address whether modified osmolytes associate with the TaMIR5062-mediated drought and salt stress responses, using transgenic lines (Sen 1, Sen 2, and Anti 1) and WT after stress treatments as samples. As expected, no variations were found on contents of soluble sugar and protein in transgenic and WT plant under normal growth condition (Figs. 5a and 5b). Under treatments of drought and salt stress, higher levels of soluble sugar and soluble protein were found in lines overexpressing TaMIR5062 than WT treated with above stressors. In contrast, the contents of soluble sugar and protein were decreased in Anti 1 compared with WT under drought and salt treatments (Figs. $5 a$ and 5b). These results suggested that TaMIR5062 modifies osmotic stress response partly by its role in regulating accumulation of the osmolytes in plants.

\section{Behaviors of ROS-associated parameters in transgenic lines under drought and salt treatments}

The ROS-associated parameters in transgenic and WT plants were measured after the drought and salt treatments. Under normal growth condition, comparable behaviors on antioxidant enzyme (AE) activities and over-oxidation product on membrane system were observed among the transgenic lines and WT plants (Figs. 6a to 6e). Under drought and salt stress treatments, compared with WT, Sen 1 and Sen 2 were enhanced on activities of SOD, CAT, and POD, decreased on contents of MDA, and lowered on membrane electric conductance (MEC) whereas Anti 1 lowered on AE activities, increased on MDA contents, and elevated on MEC under the stress conditions (Figs. $6 \mathrm{a}$ to $6 \mathrm{e}$ ). These results indicated that the cellular ROS homeostasis was modified underlying TaMIR5062 regulation, namely, this miRNA member exerts positive roles in regulating cellular ROS homeostasis by which to contribute to plant adaptation to drought and salt stresses.

\section{Expression patterns of AE family genes in transgenic lines}

Expression patterns of a subset of AE family genes were analyzed in the transgenic and WT plants after drought and salt treatments to characterize the molecular processes associated with TaMIR5062mediated ROS homeostasis. Among the genes in SOD family, NtMnSOD1 modified transcripts in transgenic lines (Sen 1, Sen 2, and Anti 1) with respect to WT. Likewise, the CAT family gene NtCAT and the POD family gene NtPOD9 displayed modified expression in transgenic lines compared with those shown in WT plants (Figs. 7a to 7f). Of which, all of the differentially expressed genes in AE families were significantly upregulated in lines overexpressing TaMIR5062 (i.e., Sen 1 and Sen 2) and downregulated in line with knockdown expression of the miRNA (Anti 1) (Figs. 7a to 7f). These results together suggest that distinct genes in AE families, including NtMnSOD1, NtCAT, and NtPOD9, respond to drought and salt signaling underlying regulation of TaMIR5086 at the transcriptional level. They contribute to the improved cellular ROS homeostasis in TaMIR5062 overexpression lines under the osmotic stress conditions. 


\section{The transcriptome profile modified by TaMIR5062 under drought stress condition}

Transcriptome profile upon drought stress underlying modulation of TaMIR5062 was investigated at global level based on RNA-seq analysis, using the drought challenged line (Sen 1) and WT as samples. In total of 3126 genes with differentially expressed (DE), including 1650 with patterns of upregulation and 1467 of downregulation, were identified (Fig. 8a). Gene ontology (GO) analysis on upregulated DE genes suggested that they are enriched into GO terms associated with "biological process", "cellular components", and "molecular function", especially that of the first one. The DE genes in GO term "biological process" associates with processes of photosynthesis, oxidation-reduction process, metabolic process, single-organism metabolic process, organonitrogen, organonitrogen compound metabolic process, organonitrogen compound biosynthetic process, protein folding, isoprenoid metabolic process, isoprenoid biosynthetic process, lipid metabolic process, oxoacid metabolic process, organic acid metabolic process, lipid biosynthesis process, small molecule metabolic process; the DE genes in GO term "cellular components" impacts on constitutions of photosystem, photosynthetic membrane, thylakoid, thylakoid part, photosystem II oxygen evolving complex, thylakoid membrane, photosystem II, photosystem I, oxidoreductase complex, photosystem I reaction center, extrinsic component of membrane, membrane protein complex; the DE genes in GO term "molecular function" exerts role in regulation of oxidoreductase activity, cofactor binding, coenzyme binding, and fructose-biophosphate aldolase activity (Fig. 8b). KEGG analysis on the DE genes identified in the drought-challenged TaMIR5062 overexpression lines suggests the modified biochemical pathways under the control of miRNA, including metabolic pathways, biosynthesis of secondary metabolites, carbon metabolism, carbon fixation in photosynthetic organisms, photosynthesis, phenylpropanoid biosynthesis, phenylalanine metabolism, glyoxylate and dicarboxylate metabolism, nitrogen metabolism, etc. (Fig. 8c). These findings together suggested that a large set of genes in plants are underlying regulation of TaMIR5062 at transcriptional level, which are involved in modifying diverse physiological processes associated with plant response to osmotic stress.

\section{Discussion}

The members of miRNA families involve modulation of diverse physiological processes of growth, development, and stress responses through regulating target genes via posttranscriptional cleavage or translation repression mechanisms (Ayushi et al. 2015). In this study, our prediction analysis on TaMIR5062, a miRNA member in T. aestivum, indicated that it interacts with six target genes. Of which, two encode calmodulins, three code for 4-oxalocrotonate tautomerases, and one encodes pumilio-family RNA binding domain protein. Thus far, extensive investigations have been performed to validate the function of genes encoding calmodulin, a critical component of the signaling pathways sensing modified levels of $\mathrm{Ca}^{2+}$ at cellular or subcellular level, in modulating wide range of stress defensive-associated biological processes (Scholz et al. 2015; Wang et al. 2016; Kim et al. 2017; Raina et al. 2021). Likewise, distinct members of RNA binding domain protein are involved in plant stress response through modulating assembly, activity, and efficiencies of transcription and translation of mRNA members 
involving plant acclimation to various abiotic stressors (Mahmood et al. 2020; Muthusamy et al. 2021; Raina et al. 2021). Therefore, most of the target genes encoding signaling and regulation-associated proteins underlying TaMIR5062 regulation suggested that this miRNA impacts on modulation of diverse biological processes, given the wide range of function mediated by target genes. Further characterization on the target genes of TaMIR5062 can help to establish the function module (i.e., TaMIR5062/calmodulin) underlying this miRNA and its target genes.

Similar to that of mRNA molecules, the transcription mechanism of miRNA members in plants species is comprehensively regulated by a subset of regulatory factors, such as enzyme Pol II proteins recruiting to promoter region of miRNA via actions of a set of transcriptional coactivators (Kim et al. 2011), TATA box acting as conserved motif involving transcription that is situated at promoter region (Xie et al. 2005), and the cis-acting regulatory elements locating at distinct positions of promoter involving DNA-protein interaction (Megraw et al. 2006; Hajdarpašić and Ruggenthaler 2012; Liang et al. 2012). To date, a subset of miRNA members in plant species has been documented with modified transcription efficiency upon osmotic stresses (Leung and Sharp 2010). In this study, our expression analysis on TaMIR5062 revealed that the transcripts of this miRNA modify sensitively upon osmotic stresses (i.e., drought and salt stress), displaying significantly upregulated under stress treatments and gradually recovered in aerial and root tissues along with normal recovery condition. These results suggest that TaMIR5062 is response to drought and salt stresses at transcription level, whose modified transcripts upon stresses impact on modulation of physiological processes via its role in regulation of its target genes. Previously, a cisregulatory motif referred to as CRE (with motif CCGCGT, CACGTGT, and AAGTCAA) present frequently in gene promoters has been verified to playing critical roles in regulating transcription efficiency of the osmotic stress-responsive genes, via an interaction mechanism between the motif with the DNA binding domain harboring bZIP transcription factors (Ma et al. 2012). Therefore, further characterization on the cis-regulatory elements, such as CRE and other ones exerting roles in modulating miRNA transcription under drought and salt stresses, can provide insights into novel understanding for transcription mechanisms of miRNA members of plant species in response to osmotic stresses.

miRNA members have been documented in mediating plant adaption to various abiotic stresses. For example, member miR319 in Arabidopsis displayed increased transcripts upon drought stress. The transgenic lines overexpressing miR319 conferred plants improved growth traits and enhanced drought tolerance by regulating leaf wax biosynthesis and water retention capacity, given that it downregulates expression of target genes AsPCF5, AsPCF6, AsPCF8, and AsTCP14, the members in TCP transcription factor family ( $\mathrm{Nag}$ et al. 2009). In this study, our transgene analysis on TaMIR5062 confirmed its positive function in mediating adaptations to drought and salt stresses, given findings that the lines overexpressing this miRNA improved drastically phenotypes, plant biomass, and photosynthetic function under the osmotic stress conditions. Therefore, our results suggested that TaMIR5062 can be used as a useful molecular index for evaluation of drought and salt adaptation in T. aestivum. It is also a valuable target for molecular breeding of the wheat cultivars to be elite in drought and salt tolerance. 
Stomata movement sensitively responds to environmental cues, which acts as one of the critical adaptation mechanisms of plants to osmotic stress conditions (Kollist et al. 2014). In this study, our analysis on stomata closing rates (SCR) in lines with overexpression or knockdown expression of TaMIR5062 revealed that the miRNA exerts large role in regulating stomata movement; the lines overexpressed the miRNA (i.e., Sen 1 and Sen 2) displayed faster on decrease of SCR whereas that with knockdown expression of miRNA (Anti 1) slower on SCR reduction under treatments of drought and salt stress, with respect to WT plants. Additionally, TaMIR5062 also confers plants enhanced osmo-regulatory ability, based on our osmolytes assay results in the stress-challenged transgenic lines. These results suggested that TaMIR5062 functions as a mediator in improving osmotic stress tolerance through the mechanism to enhance water retention capacity via regulating stomata movement and osmolyte biosynthesis. Thus far, extensive literatures have reported the internal relation between stomata movement upon osmotic stressors and the induced abscisic acid (ABA) accumulation (Boursiac et al. 2013; Song et al. 2014). Moreover, the ABA signaling pathways associated with stomata movement under stress signaling, such as ABA receptors (i.e., RCAR, PYR1, and PYL), type 2C protein phosphatases (PP2C), and SNF1-related protein kinase OPEN STOMATA1 (OST1)/SnRK2 that function as essential members in pathways, have been extensively characterized in the model plants (Park et al. 2009; Ma et al. 2009). Therefore, further investigation of the ABA signaling pathway regulating stomata movement underlying TaMIR5082 modulation can provide insights into plant osmotic stress responses mediated by miRNA members.

Plant tissues display induced ROS upon abiotic stresses, which negatively impact on plant stress adaptations due to its injury on molecule structures of protein, lipid, and nucleic acid and cell function (Gill and Tuteja 2010). Acting as effective agents for ROS scavenging, the AE enzymes SOD, CAT, and POD alleviate osmotic stress effects and contribute to cellular ROS homeostasis (You and Chan 2015). For example, overexpression of $N$. plumbaginifolia MnSOD gene in alfalfa significantly improved growth traits and yield potential of plants treated with drought stress (McKersie et al. 1996). The O. sativa lines overexpressing A. marina cytosolic CUZnSOD display enhanced ability to osmotic stress (Prashanth et al. 2008). In this study, our assay on SOD, CAT, and POD activities in lines with TaMIR5062 overexpression or knockdown expression validated the improvement and alleviation of them under osmotic stress conditions, which impact on behavior of contents of MDA, membrane electric conductance (MEC), and finally cellular ROS homeostasis. Moreover, based on expression analysis, we identified distinct genes to be differentially expressed in the TaMIR5062 overexpression line and the line with miRNA knockdown expression, including SOD gene NtMnSOD1, CAT gene NtCAT, and POD gene NtPOD9 under stress treatments. Our findings thus suggested that the TaMIR5062-mediated ROS homeostasis is associated with the modified transcription of distinct AE genes mentioned above that enhance cellular AE activities. Further characterization on the relations among these differential AE genes and ROS homeostasis can help the understanding of plant drought response underlying the miRNA member-ROS pathways in plant species.

High throughput RNA-seq analysis can identify genes that are modified on transcripts upon environmental stressors at global level, which provides novel understanding of the molecular processes 
as to plant stress responses (Kreps et al. 2002; Metzker 2010; Zhao et al. 2021). In this study, our RNA-seq analyses revealed that in total of 3126 genes displaying significantly modified expression (i.e., 1650 upregulated and 1476 downregulated) in the drought-challenged lines with TaMIR5062 overexpression. That the DE genes are categorized into diverse GO terms referred to as "biological process", "cellular components", and "molecular function" and they are overrepresented by a subset of biochemical pathways suggest the complicate nature in miRNA-mediated plant drought and salt stress response. Based on GO terms enriched and KEGG pathways overrepresented by the drought upregulated DE genes, we depicted a diagram to illustrate plant responses to osmotic stress (i.e., drought) underlying TaMIR5062 regulation (Fig. 9). TaMIR5062 mediates plant drought adaptation to be accomplished through three sequential biochemical pathways: (i) the pathway is constituted by biological process (G0:0008150), cellular process (G0:0009987), and protein folding (G0:0006457); (ii) pathways are consisted of biological process (G0:0008150), signal-organism process (G0:0044699), cellular lipid metabolism (G0:0044255), isoprenoid metabolic process (GO:0006720), which impact on isoprenoid biosynthesis (GO: 0008299); (iii) pathways are composed of biological process (GO:0008150), metabolic process (GO:0008152) modulating cellular metabolic process (G0:0044237), biosynthetic process (G0:0009058), signal-organism metabolic process (G0:0044710), primary metabolic process (GO: 0044238), organic substance metabolic process (G0:0071704), and nitrogen compound metabolic process (G0:0006807); these processes further modulate following biochemical metabolisms, including cellular biosynthesis metabolism (G0:0044249), signal-organism biosynthesis metabolism (G0:0044711), oxidation-reduction metabolism (G0:0055114), lipid metabolic process (G0:0006629), organic substance biosynthesis metabolism (GO:1901576), and oranotrogen compound metabolism (GO:1901564), and finally the isoprenoid biosynthesis metabolism (GO:0008299). In addition, for the third pathway, an important one that involves photosynthetic function modulation needs to be mentioned, which is constituted by biological process (G0:0008150), metabolic process (G0:0008152), cellular metabolic process (G0:0044237), photosynthesis (G0:0015979). These metabolism- and biosynthesisassociated biological processes modulated by DE genes comprehensively impact on water retention, ROS homeostasis, and plant osmotic stress response underlying the miRNA regulation

\section{Conclusion}

TaMIR5062 targets six genes that are functional in various biological processes. The transcripts of TaMIR5062 were upregulated upon drought and salt stress conditions. TaMIR5062 conferred plants improved drought and salt adaptations, which are closely associated with its function in modulating water retention capacity and cellular ROS homeostasis. Distinct AE genes, including SOD family member NtMnSOD1, CAT family member NtCAT, and POD family one NtPOD9 that are upregulated on expression in the TaMIR5062 lines treated with drought and salt stress. TaMIR5062 modifies transcription of a large set of genes in osmotic stress-challenged plants, which improve the acclimation of plants to stress by modulating biological processes associated with distinct biological pathways (i.e., protein folding) and biosynthesis metabolisms (i.e., photosynthesis and isoprenoid biosynthesis). TaMIR5062 can be a useful 
index for evaluation of plant osmotic stress tolerance in $T$. aestivum and one valuable target for molecular breeding of elite crop cultivars for resistance of drought and salt stresses.

\section{Declarations}

\section{Acknowledgments}

This work was supported by the National Natural Science Foundation of China (No. 31872869) and Science and Technology Planning Project of Hebei Province (Local Scientific Research Project supported by Chinese government, No. 216Z6401G).

\section{Author contributions}

Kai Xiao and Chengjin Guo designed the research. Yuanjinzhi Qiao, Ling Wang, Zidi Yu, Chenyang Ni, and Tiaojiao Li conducted the experiment and performed data analysis. Kai Xiao wrote the paper.

\section{Compliance with ethical standards}

Conflict of interest All authors declare that they have no conflict of interest.

\section{Data availability statement}

All data generated or used during the study appear in the submitted article.

\section{References}

1. Ayushi K, Abira C, Mohan K, Asis D (2015) Small RNAs in plants: recent development and application for crop improvement. Front Plant Sci 6: 208

2. Bai QQ, Wang XY, Chen X, Shi GQ, Liu ZP, Guo CJ, Xiao K (2018) Wheat miRNA taemiR408 acts as an essential mediator in plant tolerance to Pi deprivation and salt stress via modulating stressassociated physiological processes. Front Plant Sci 9: 499

3. Bakhshi B, Fard EM, Gharechahi J, Safarzadeh M, Nikpay N, Fotovat R, Azimi MR, Salekdeh GH (2017) The contrasting microRNA content of a drought tolerant and a drought susceptible wheat cultivar. J Plant Physiol 216: 35-43

4. Bolger AM, Marc L, Bjoern U (2014) Trimmomatic: a flexible trimmer for Illumina sequence data. Bioinform 15: 2114-2120

5. Boyle El, Weng S, Gollub J, Jin H, Botstein D, Cherry JM, Sherlock G (2004) GO: TermFinder-open sources software for accessing Gene Ontology information and finding significantly enriched Gene Ontology terms associated with a list of genes. Bioinform 20(18): 3710-3715

6. Benjamin JG, Nielsen DC (2006) Water deficit effects on root distribution of soybean, field pea and chickpea. Field Crops Res 97: 248-253 
7. Benjamini Y, Hochberg Y (1995) Controlling the false discovery rate: A practical and powerful approach to multiple testing. J Roy Stat Soc B 57(1): 289-300

8. Boursiac Y, Léran S, Corratgé-Faillie C, Gojon A, Krouk G, Lacombe B (2013) ABA transport and transporters. Trends Plant Sci18: 325-333

9. Cattivelli L, Rizza F, Badeck FW, Mazzucotelli E, Mastrangelo AM, Francia E, Mare C, Tondelli A, Stanca AM (2008) Drought tolerance improvement in crop plants: an integrated view from breeding to genomics. Field Crops Res 105: 1-14

10. Chaves MM, Flexas J, Pinheiro C (2009) Photosynthesis under drought and salt stress: regulation mechanisms from whole plant to cell. Ann Bot (Lond) 103 (4): 551-560

11. Danquah A, de Zelicourt A, Colcombet J, Hirt H (2014) The role of ABA and MAPK signaling pathways in plant abiotic stress responses. Biotechnol Adv 32 (1): 40-52

12. Delormel TY, Boudsocq M (2019) Properties and functions of calcium-dependent protein kinases and their relatives in Arabidopsis thaliana. New Phytol 224 (2): 585-604

13. Ding D, Zhang LF, Wang H, Liu ZJ, Zhang ZX, Zheng YL (2009) Differential expression of miRNAs in response to salt stress in maize roots. Ann Bot 103: 29-38

14. Ding ZJ, Yan JY, Xu XY, Yu DQ, Li GX, Zhang SQ, Zheng SJ (2014) Transcription factor WRKY 46 regulates osmotic stress responses and stomatal movement independently in Arabidopsis. Plant $\mathrm{J}$ 79 (1): 13-27

15. Du XM, Zhao XL, Liu XM, Guo CJ, Lu WJ, Gu JT, Xiao K (2013) Overexpression of TaSRK2C1, wheat SNF1-related protein kinase gene, increases tolerance to dehydration, salt, and low temperature in transgenic tobacco. Plant Mol Biol Rep 31: 810-821

16. Fang YJ, Xiong LZ (2015) General mechanisms of drought response and their application in drought resistance improvement in plants, Cell Mol Life Sci 72(4): 673-689

17. Gill SS, Tuteja N (2010) Reactive oxygen species and antioxidant machinery in abiotic stress tolerance in crop plants. Plant Physiol Biochem 48: 909-930

18. Guo CJ, Zhao XL, Liu XM, Zhang LJ, Gu JT, Li XJ, Lu WJ, Xiao K (2013) Function of wheat phosphate transporter gene TaPHT2;1 in Pi translocation and plant growth regulation under replete and limited Pi supply conditions. Planta 237: 1163-1178

19. Hajdarpašić A, Ruggenthaler $P$ (2012) Analysis of miRNA expression under stress in Arabidopsis thaliana. Bosn J Basic Med Sci 12: 169-176

20. Haldar S, Bandyopadhyay S (2021). Co-ordinated regulation of miRNA and their target genes by CREs during salt stress in Oryza sativa (rice). Plant Gene 38: 100323

21. Hu DG, Sun CH, Ma QJ, You CX, Cheng L, Hao YJ (2016) MdMYB1 regulates anthocyanin and malate accumulation by directly facilitating their transport into vacuoles in apples. Plant Physiol 170: 13151330

22. Huang XS, Liu JH, Chen XJ (2010) Overexpression of PtrABF gene, a bZIP transcription factor isolated from Poncirus trifoliata, enhances dehydration and drought tolerance in tobacco via 
scavenging ROS and modulating expression of stress-responsive genes. BMC Plant Biol 10: 230

23. Jung H, Chung PJ, Park S, Christian M, Redillas F, Kim YS, Suh J, Kim J (2017) Overexpression of OsERF48 causes regulation of OsCML16, a calmodulin-like protein gene that enhances root growth and drought tolerance. Plant Biotechnol J 15: 1295-1308

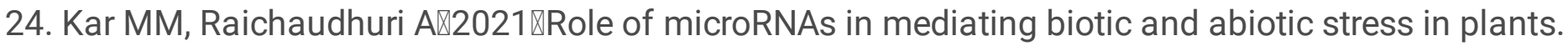
Plant Gene 26(8): 100277

25. Kim YJ, Zheng B, Yu Y, Won SY, Mo B, Chen X (2011) The role of mediator in small and long noncoding RNA production in Arabidopsis thaliana. EMBO J 30: 814-822

26. Kollist H, Nuhkat M, Roelfsema MR (2014) Closing gaps: linking elements that control stomatal movement. New Phytol203: 44-62

27. Kreps JA, Wu Y, Chang HS, Zhu T, Wang X, Harper J (2002) Transcriptome changes for Arabidopsis in response to salt, osmotic and cold stress. Plant Physiology 130: 2129-2141

28. Leung AKL, Sharp PA (2010) MicroRNA functions in stress responses, Mol Cell, 40(2): 205-215

29. Lewis R, Mendu V, Mcnear D, Tang G (2009) Roles of microRNAs in plant abiotic stress. In: SM Jain, DS Brar, eds, Molecular Techniques in Crop Improvement, 2nd edn. Springer, Cambridge, pp 357-372

30. Liang G, He H, Yu D (2012) Identification of nitrogen starvation-responsive microRNAs in Arabidopsis thaliana. PLoS One 7: e48951

31. Liu HH, Tian X, Li YJ, Wu CA, Zheng CC (2008) Microarray-based analysis of stress-regulated microRNAs in Arabidopsis thaliana. RNA, 14(5): 836-843

32. Lu W, Li J, Liu F, Gu J, Guo C, Xu L, Zhang H, Xiao Kai (2011) Expression pattern of wheat miRNAs under salinity stress and prediction of salt-inducible miRNAs targets. Front Agric China 5: 413-422

33. Lu XY, Huang XL (2008) Plant miRNAs and abiotic stress responses. Biochem Biophys Res Commun 368: $458-462$

34. Lv DK, Bai X, Li Y, Ding XD, Ge Y, Cai H, Ji W, Wu N, Zhu YM (2010) Profiling of cold-stress-responsive miRNAs in rice by microarrays. Gene 459: 39-47

35. Lv SZ, Nie XJ, Wang L, Du XH, Biradar SS, Jia XO, Song WN (2012) Identification and characterization of microRNAs from barley (Hordeum vulgare L.) by high-throughput sequencing. Int J Mol Sci 13: 2973-2984

36. Ma S, Bachan S, Porto M, Bohnert HJ, Snyder M, Dinesh-Kumar SP (2012) Discovery of stress responsive DNA regulatory motifs in Arabidopsis. PLoS One 7: e43198

37. Ma Y, Szostkiewicz L, Korte A, Moes D, Yang Y, Christmann A, Grill E (2009) Regulators of PP2C phosphatase activity function as abscisic acid sensors. Science324: 1064-1068

38. Mahmood T, Khalid S, Abdullah M, Ahmed Z, Kausar MNS, Ghafoor A, Du X (2020) Insights into drought stress signaling in plants and the molecular genetic basis of cotton drought tolerance. Cells 9: 105

39. Muthusamy M, Kim J, Kim JA, Lee S (2021) Plant RNA binding proteins as critical modulators in drought, high salinity, heat, and cold stress responses: an updated overview. Int J Mol Sci 22: 6731 
40. Kar MM, Raichaudhuri A (2021) Role of microRNAs in mediating biotic and abiotic stress in plants. Plant Gene 26: 100277

41. Maiti RK, Satya P (2014) Research advances in major cereal crops for adaptation to abiotic stresses, GM Crops Food 5(4): 259-279

42. Mazzucotelli E, Mastrangelo AM, Crosatti C, Guerra D, Stanca AM, Cattivelli L (2008) Abiotic stress response in plants: when post-transcriptional and post-translational regulations control transcription. Plant Sci 174: 420-431

43. McKersie BD, Bowley SR, Harjanto E, Leprince O (1996) Water-deficit tolerance and field performance of transgenic alfalfa overexpressing superoxide dismutase. Plant Physiol 111: 1177-1181

44. Megraw M, Baev V, Rusinov V, Jensen ST, Kalantidis K, Hatzigeorgiou AG (2006) MicroRNA promoter element discovery in Arabidopsis. RNA 12: 1612-1619

45. Metzker ML (2010) Sequencing technologies the next generation. Nat Rev Genet 11(1): 31-46

46. Nag A, King S, Jack T (2009) miR319a targeting of TCP4 is critical for petal growth and development in Arabidopsis. Proc Natl Acad Sci USA 106: 22534-22539

47. Naheed R, Aslam H, Kanwal H, et al. (2021) Growth attributes, biochemical modulations, antioxidant enzymatic metabolism and yield in Brassica napus varieties for salinity tolerance. J Biol Sci 28(10): $5469-5479$

48. Ori N, Cohen AR, Etzioni A, et al. (2007) Regulation of Lanceolate by miR319 is required for compound-leaf development in tomato. Nat Genet 39: 787-791

49. Palatnik JF, Allen E, Wu X, Schommer C, Schwab R, Carrington JC, Weigel D (2003) Control of leaf morphogenesis by microRNAs. Nature 425: 257-263

50. Park Fung P, Nishimura N, R.Jensen D, et al. (2009) Abscisic acid inhibits type $2 \mathrm{C}$ protein phosphatases via the PYR/PYL family of start proteins. Science324: 1068-1071

51. Phillips JR, Dalmay T, Bartels D (2007) The role of small RNAs in abiotic stress. FEBS Lett 581: 3592-3597

52. Prashanth SR, Sadhasivam V, Parida A (2008) Over expression of cytosolic copper/zinc superoxide dismutase from a mangrove plant Avicennia marina in indica rice var Pusa Basmati-1 confers abiotic stress tolerance. Transgenic Res 17: 281-291

53. Qiu CW, Liu L, Feng X, Hao PF, He XY, Cao FB, Wu FB (2020) Genome-wide identification and characterization of drought Stress responsive microRNAs in Tibetan wild barley. Int J Mol Sci 21(8): 2795

54. Raina M, Kisku AV, Joon S, Kumar S, Kumar D (2021) Calcium transport elements in plants. In: Upadhyay SK Calmodulin and calmodulin-like $\mathrm{Ca}^{2+}$ binding proteins as molecular players of abiotic stress response in plants. Salt Lake City, American Academic Press, pp 231-248

55. Read S M, Northcote D H (1981) Minimization of variation in the response to different protein of the Coomassie blue $G$ dye-binding: assay for protein. Anal Biochem 116(1): 53-64 
56. Robinson MD, McCarthy DJ, Smyth GK (2010) edgeR: a Bioconductor package for differential expression analysis of digital gene expression data. Bioinformatics 26(1): 139-140

57. Rubio-Somoza I, Weigel D (2011) MicroRNA networks and developmental plasticity in plants. Trends Plant Sci 16: 258-264

58. Scholz SS, Reichelt M, Vadassery J, Mithöfer A (2015) Calmodulin-like protein CML37 is a positive regulator of ABA during drought stress in Arabidopsis. Plant Signal Behav 10: e1011951

59. Shi MH, Wang Z, Ma ZF, Song WT, Lu WJ, Xiao K (2020) Characterization on TaMPK14, an MAPK family gene of wheat, in modulating $\mathrm{N}$-starvation response through regulating $\mathrm{N}$ uptake and ROS homeostasis, Cell Reports 39(10): 1285-1299

60. Shukla LI, Chinnusamyb V, Sunkara R (2008) The role of microRNAs and other endogenous small RNAs in plant stress responses. BBA-Gene Regul Mech 1779(11): 743-748

61. Sirichandra C, Gu D, Hu HC, et al. (2009) Phosphorylation of the Arabidopsis AtrbohF NADPH oxidase by OST1 protein kinase. Febs Lett 583: 3375-3375

62. Song XJ, Matsuoka M (2009) Bar the windows: an optimized strategy to survive drought and salt adversities. Genes \& Dev 23: 1709-1713

63. Song YW, Miao YC, Song CP (2014) Behind the scenes: the roles of reactive oxygen species in guard cells. New Phytol201: 1121-1140

64. Sun F, Guo G, Du J, Guo W, Peng H, Ni Z, Sun Q, Yao Y (2014) Whole-genome discovery of miRNAs and their targets in wheat (Triticum aestivum L.). BMC Plant Biol 14: 142

65. Sun Z, Ding C, Li X, Xiao K (2012). Molecular characterization and expression analysis of TaZFP15, a $\mathrm{C} 2 \mathrm{H} 2$ - type zinc finger transcription factor gene in wheat (Triticum aestivum L.). J Integr Agric 11: $31-42$

66. Sun X, Lin L, Sui N (2019). Regulation mechanism of microRNA in plant response to abiotic stress and breeding. Mol Biol Rep 46(1): 1447-1457

67. Sunkar R, Zhu JK (2004) Novel and stress-regulated microRNAs and other small RNAs from Arabidopsis. Plant Cell 16: 2001-2019

68. Terletskaya $N \rrbracket K$ Khailenko $N$, Zhambakin $K \otimes 2013 \llbracket$ Stability of cereal crops to drought and saline stress in vivo and in vitro. J Life Sci (USA) 7(2): 135-144

69. Thiebaut F, Rojas CA, Almeida KL, Grativol C, Domiciano GC, Lamb CRC, Engler JA, Hemerly AS, Ferreira PCG (2012) Regulation of miR319 during cold stress in sugarcane. Plant Cell Environ 35: $502-512$

70. Wang B, Sun YF, Song N, Wei JP, Wang XJ, Feng H, Yin ZY, Kang ZS (2014) MicroRNAs involving in cold, wounding and salt stresses in Triticum aestivum $L$. Plant Physiol Biochem 80: 90-96

71. Wang X, Cai X, Xu C, Wang Q, Dai S (2016) Drought-responsive mechanisms in plant leaves revealed by proteomics. Int J Mol Sci 17: 1706

72. Xie Z, Allen E, Fahlgren N, Calamar A, Givan SA, Carrington JC (2005) Expression of Arabidopsis MIRNA genes. Plant Physiol 138: 2145-2154 
73. Yang YQ, Guo Y (2018) Elucidating the molecular mechanisms mediating plant salt-stress responses, New Phytol 217(2): 523-539

74. Yoshida T, Mogami J, Yamaguchi-Shinozaki K (2014) ABA-dependent and ABA-independent signaling in response to osmotic stress in plants. Curr Opin Plant Biol 21: 133-139

75. You J, Chan Z (2015) ROS regulation during abiotic stress responses in crop plants. Front Plant Sci 6: 1092

76. Zhang XY, Xu MY, Zheng HY, Zou JJ, Zhang XY, Wang L (2020) Analysis of miRNA expression profiles in rice under salt stress. J Agr Sci Tech 22(12): 10-19

77. Zhao BT, Ge LF, Liang RQ, Li W, Ruan KC, Lin HX, Jin YX (2009) Members of miR-169 family are induced by high salinity and transiently inhibit the NF-YA transcription factor, BMC Mol Bio 10: 2938

78. Zhao JM, Pan L, Zhou M, Yang ZF, Meng Y, Zhang XQ (2019) Comparative physiological and transcriptomic analyses reveal mechanisms of improved osmotic stress tolerance in annual ryegrass by exogenous chitosan. Genes (Basel) 10(11): 853


throughput illumina strand-specific RNA sequencing library preparation. Cold Spring Harbor Protocols 8: 940-949

80. Zhao SS, Zhang QK, Liu MY, Zhou HP, Ma CL, Wang PP (2021) Regulation of Plant Responses to Salt Stress. Int J Mol Sci 22(9): 4609

81. Zhou L, Liu Y, Liu Z, Kong D, Duan M, Luo L (2010) Genome-wide identification and analysis of drought-responsive microRNAs in Oryza sativa. J Exp Bot 61: 4157-4168.

\section{Figures}




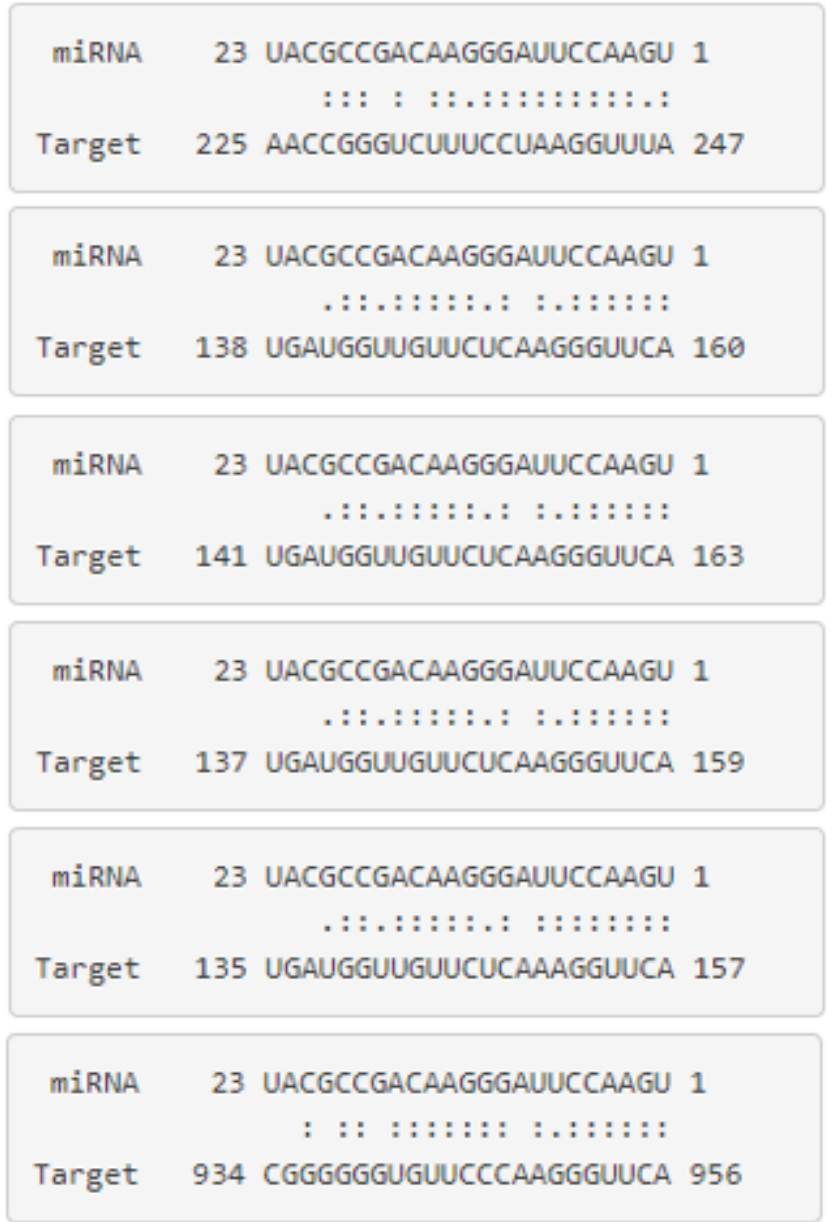

\author{
TaMIR5062 (MI0030391) \\ calmodulin (CA690003) \\ TaMIR5062 (MI0030391) \\ calmodulin (CA700147) \\ TaMIR5062 (MI0030391) \\ 4-oxalocrotonate tautomerase (CA593090) \\ TaMIR5062 (MI0030391) \\ 4-oxalocrotonate tautomerase (CA679560) \\ TaMIR5062 (MI0030391) \\ 4-oxalocrotonate tautomerase (CA484824) \\ TaMIR5062 (MI0030391) \\ pumilio-family RNA binding domain ( $\mathrm{CK} 171376$ )
}

\title{
Figure 1
}

Target genes of TaMIR5062 and the sequence base pairing characterization among them

Six genes are shown to share high base pairing nature with TaMIR5062, including two coding for calmodulins (No. CA690003 and No. CA700147), three encoding 4-oxalocrotonate tautomerases (No. CA593090, No. CA679560, and No. CA484824), and one encoding a pumilio-family RNA binding domain protein (No. CK171376) 


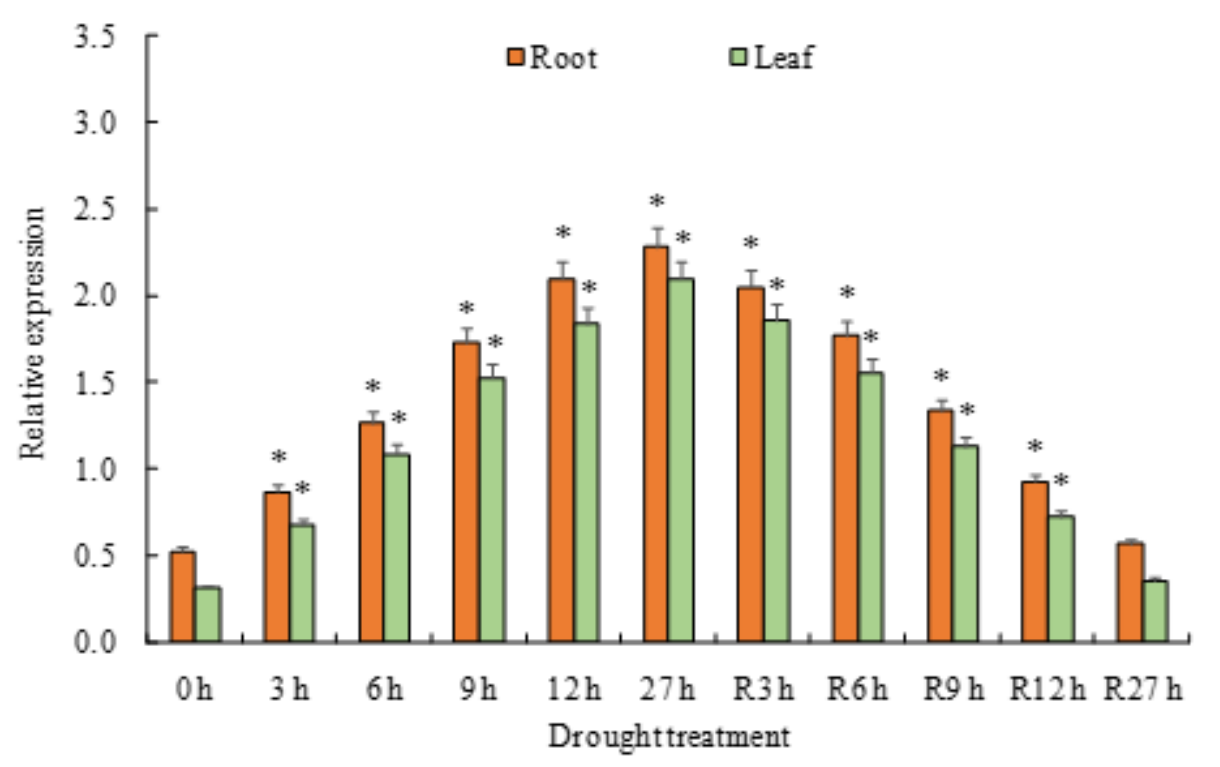

b

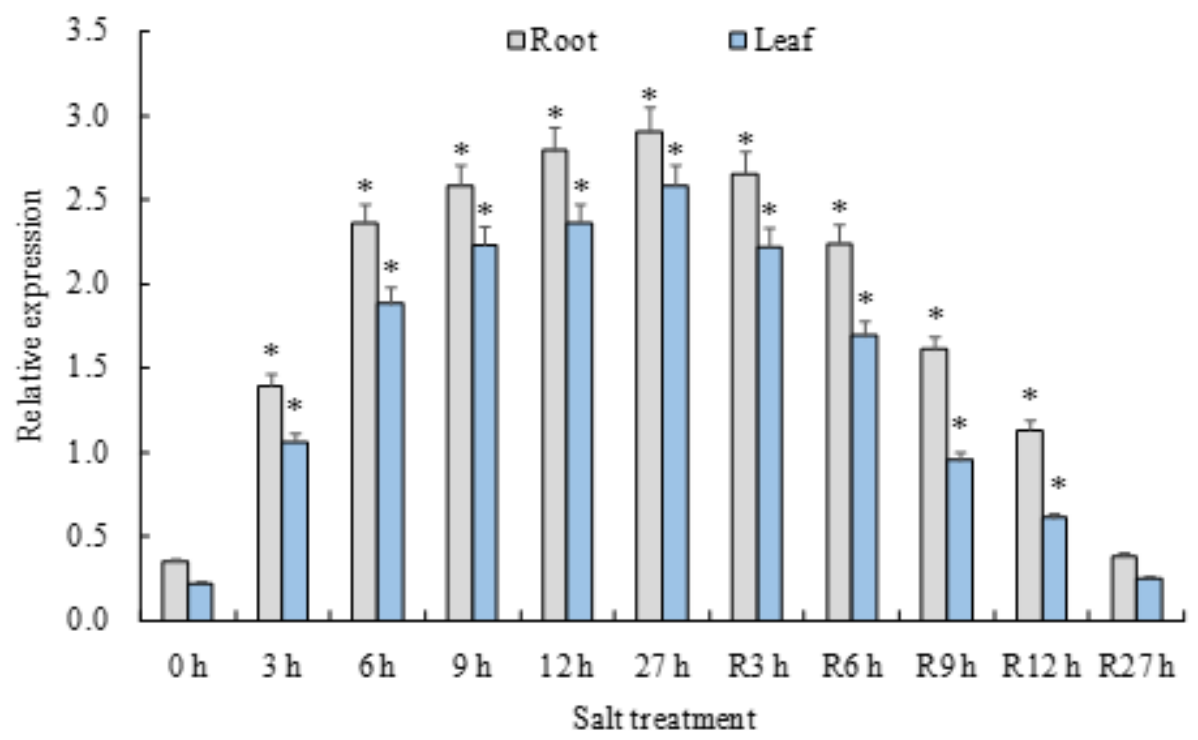

Figure 2

Expression patterns of TaMIR5062 upon drought and salt stress treatments

a, relative expression under drought stress condition; $\mathbf{b}$, relative expression under salt stress condition. 0 h, time prior to treatment; 3 h, 6 h, 9 h, 12 h, and 27 h, time points during drought and salt treatments; R3 $h, R 6 h, R 9 h, R 12 h$, and R27 h, time points during the normal recovery treatment. Data are normalized by 
internal standard Tatubulin and shown by averages plus the standard errors with symbol * to represent statistically significant at indicated times with respect to $0 \mathrm{~h}(\mathrm{P}<0.05)$.
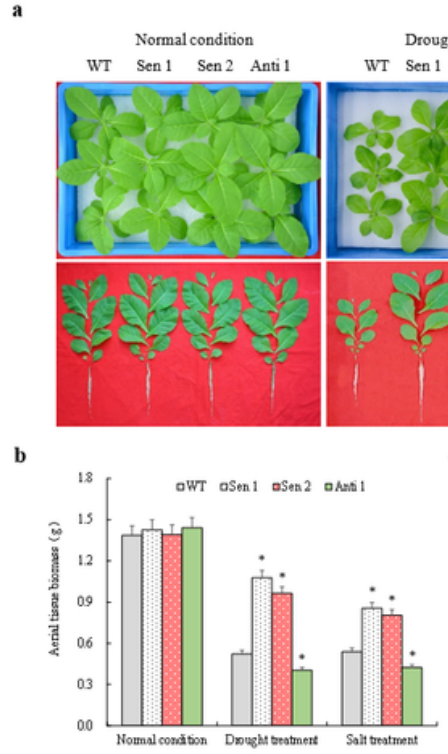
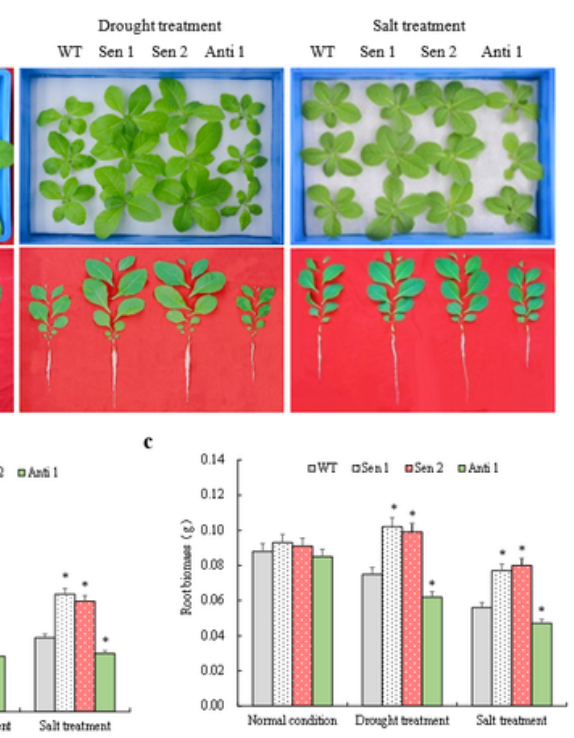

Salt treatment

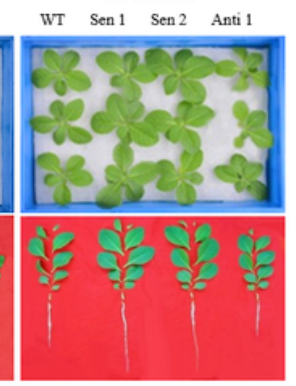

f
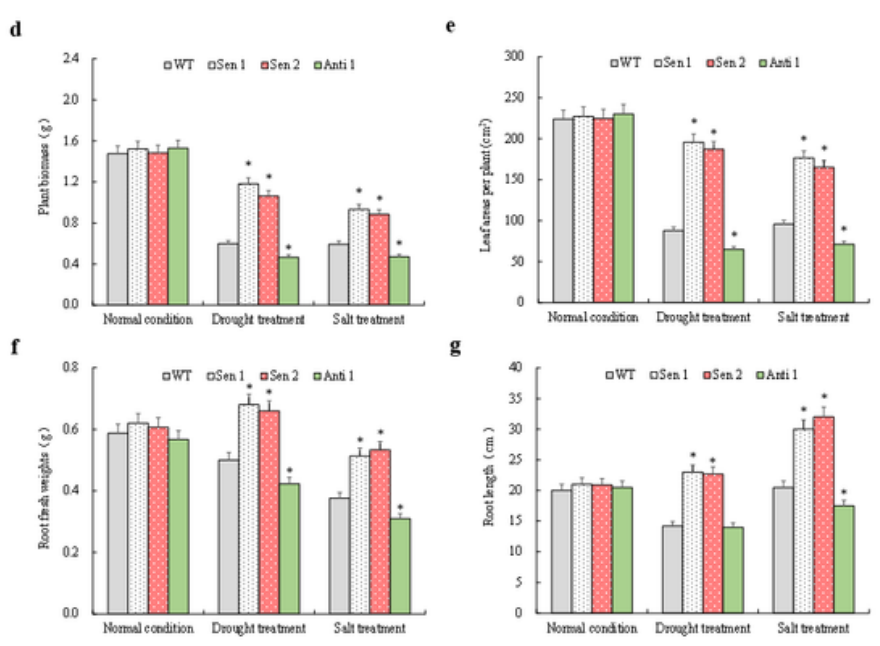

\section{Figure 3}

Phenotypes and growth traits in transgenic lines of TaMIR5062 under drought and salt treatments

a, phenotypes under normal condition, drought, and salt stress conditions; $\mathbf{b}$, aerial tissue biomass; $\mathbf{c}$, root biomass; $\mathbf{d}$, plant biomass; e, leaf areas; $\mathbf{f}$, root fresh weights; $\mathbf{g}$, root lengths. WT, wild type; Sen 1 and Sen 2, two lines overexpressing TaMIR5062. Anti 1, one line with knockdown expression of TaMIR5062. In b to g, data shown are average plus standard error with symbol * to represent statistically significant compared with WT $(P<0.05)$ 
a

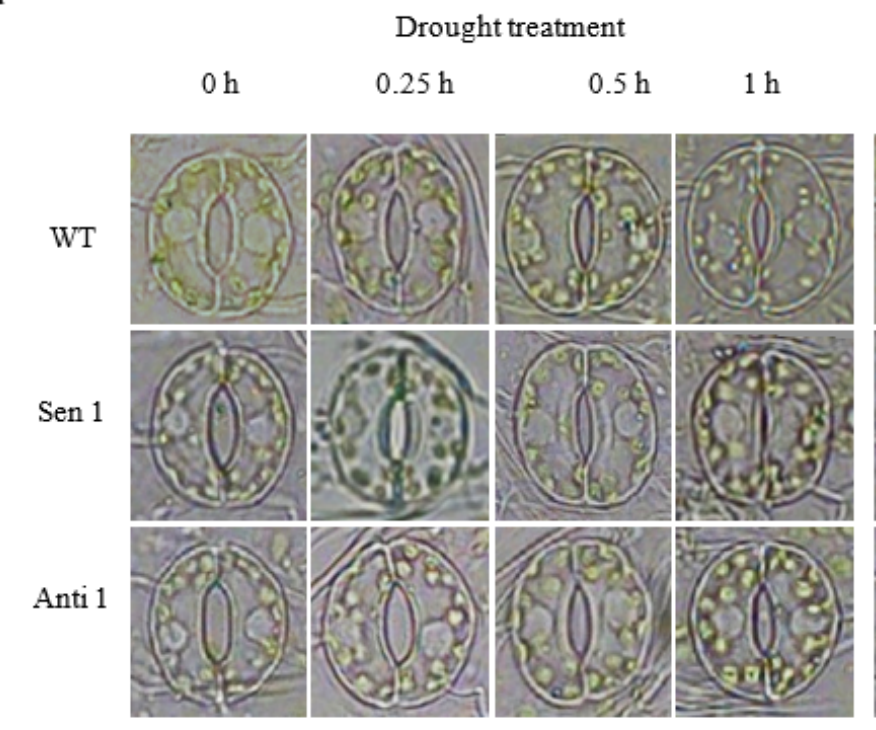

b



d

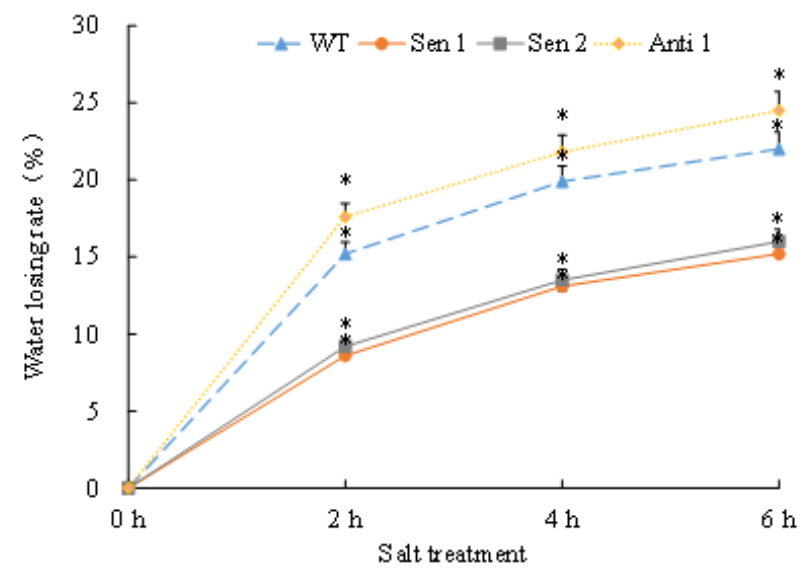

\section{Figure 4}

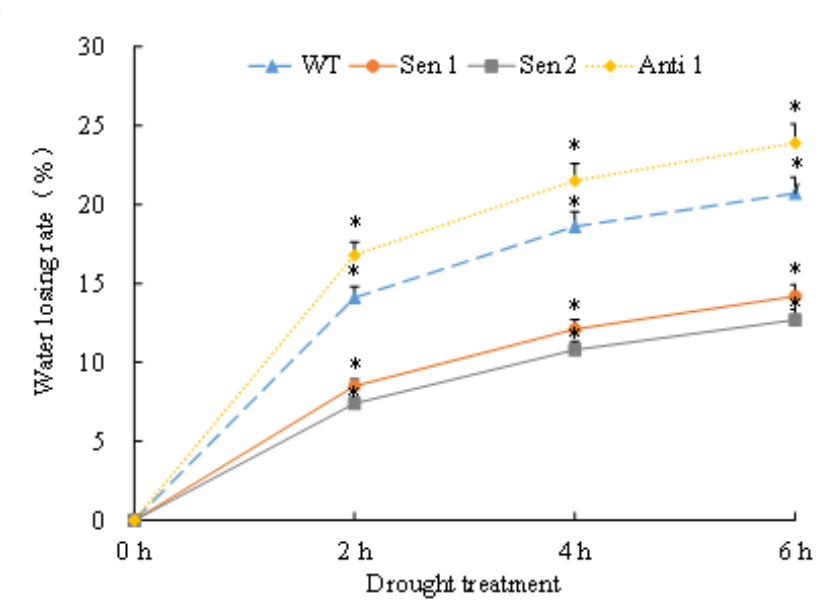

Stomata aperture and water retention capacity of leaves in transgenic lines upon drought and salt treatments

a, stomatal aperture rates under drought stress condition; $\mathbf{b}$, stomatal aperture rates under salt stress condition; c, water losing rates under drought stress condition; $\mathbf{d}$, water losing rates under salt condition. 0 $\mathrm{h}$, time point for leaves immediately collected; $0.25 \mathrm{~h}, 0.5 \mathrm{~h}$, and $1 \mathrm{~h}$, time points for leaves detached from plants for water deficit treatment. WT, wild type; Sen 1 and Anti 1, two transgenic lines with TaMIR5062 overexpression and knockdown expression, respectively. In $\mathbf{c}$ and $\mathbf{d}$, data shown are average plus standard error with symbol * to represent statistically significant compared with WT $(P<0.05)$. 
a

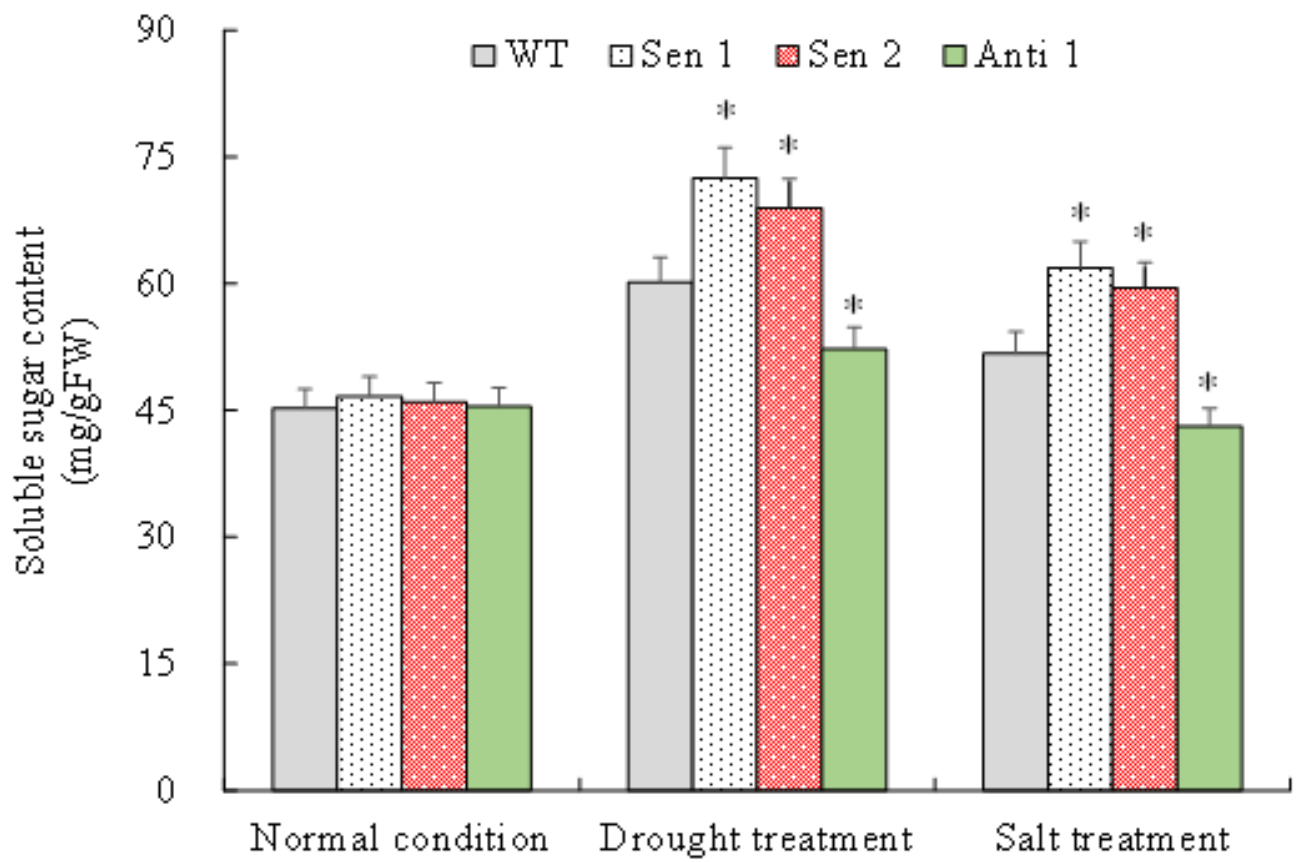

b

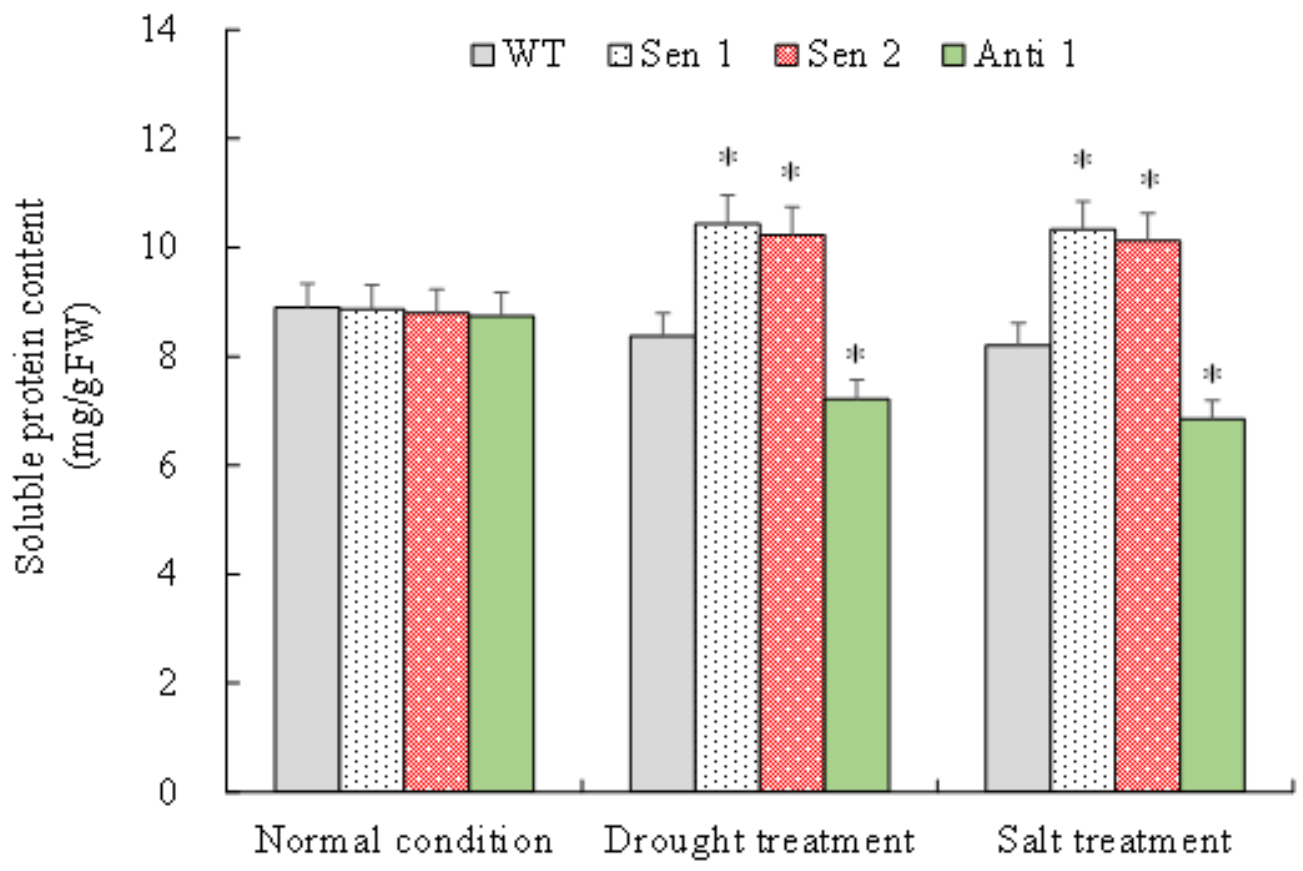

Figure 5

Contents of soluble sugar and soluble protein in the transgenic lines under drought and salt treatments

a, soluble sugar contents; b, soluble protein contents. WT, wild type; Sen 1 and Sen 2, two transgenic lines overexpressing TaMIR5062; Anti 1, one line with knockdown expression of TaMIR5062. Data shown are 
average plus standard error with symbol * to represent statistically significant compared with WT $(P<0.05)$

a

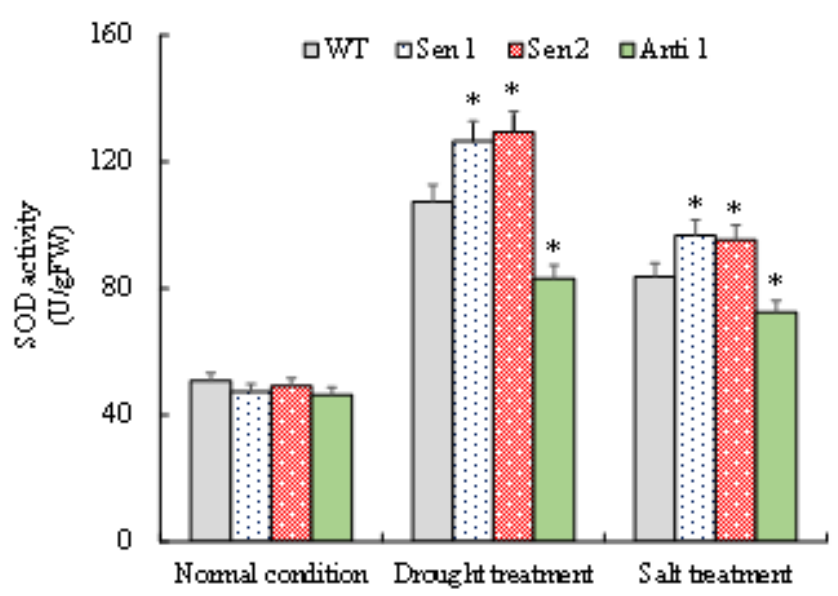

$\mathbf{c}$

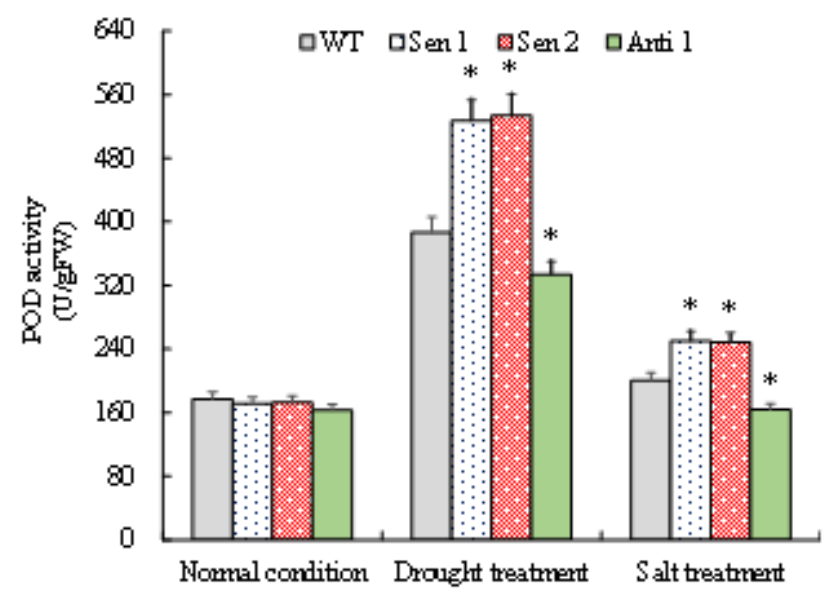

e

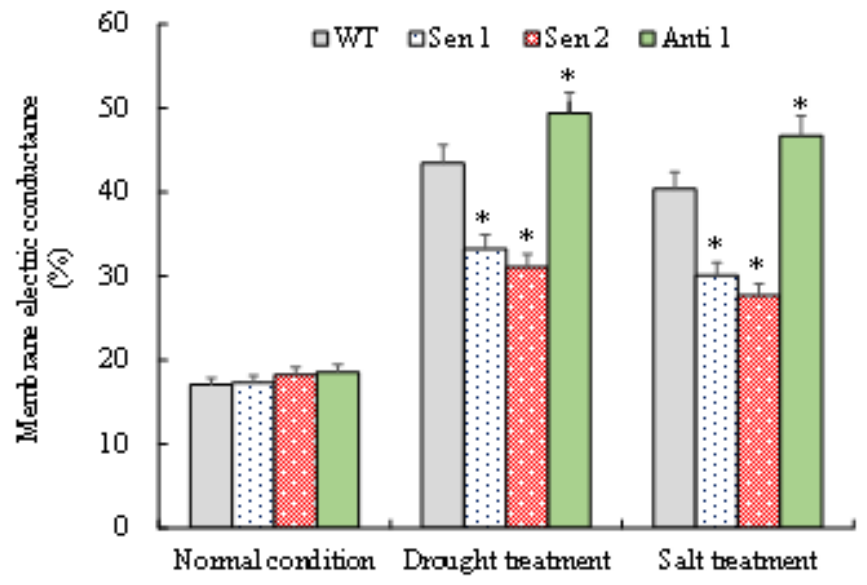

b

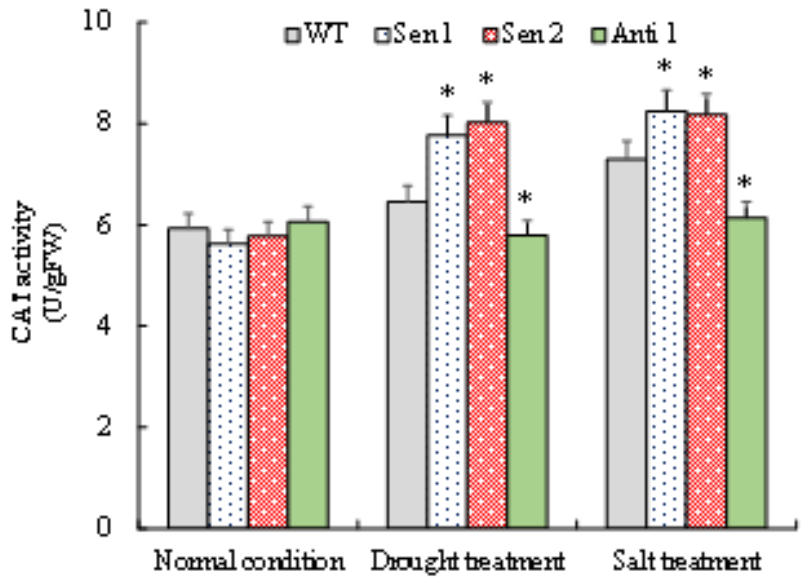

d



Figure 6

Cellular ROS-associated parameters and membrane electric conductance in the transgenic lines under drought and salt treatments 
a, SOD activities; b, CAT activities; c, POD activities; d, MDA contents; e, membrane electric conductance. WT, wild type; Sen 1 and Sen 2, two transgenic lines overexpressing TaMIR5062; Anti 1, one line with knockdown expression of TaMIR5062. Data shown are average plus standard error with symbol * to represent statistically significant compared with WT $(P<0.05)$

a

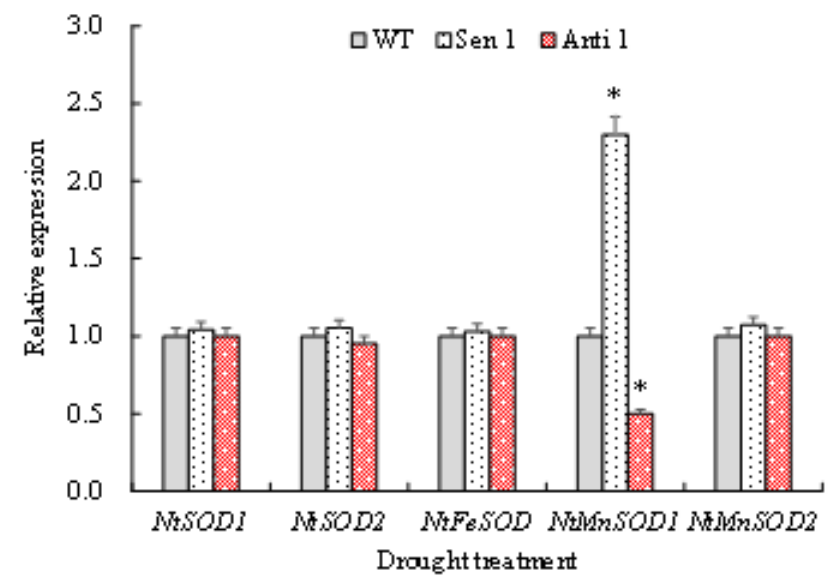

C

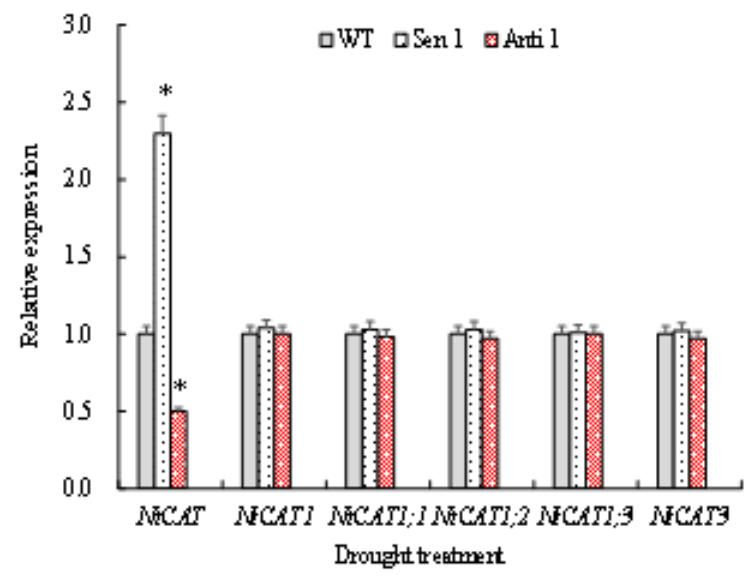

e

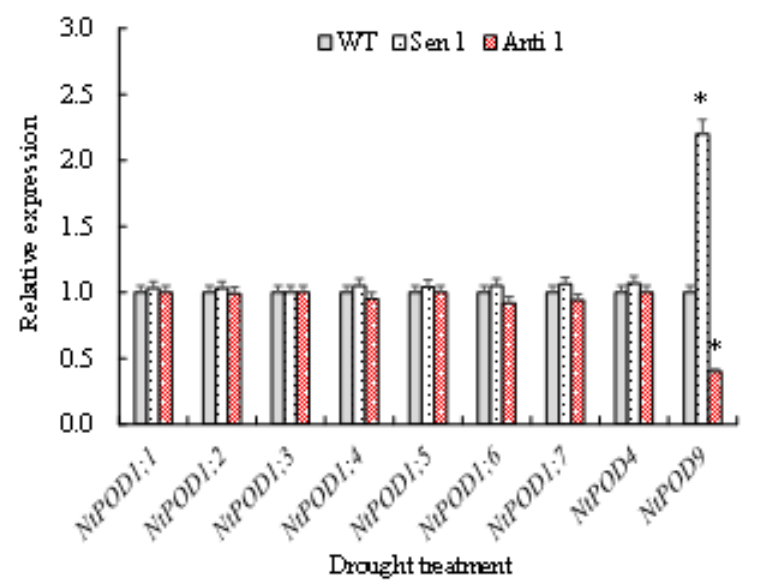

b

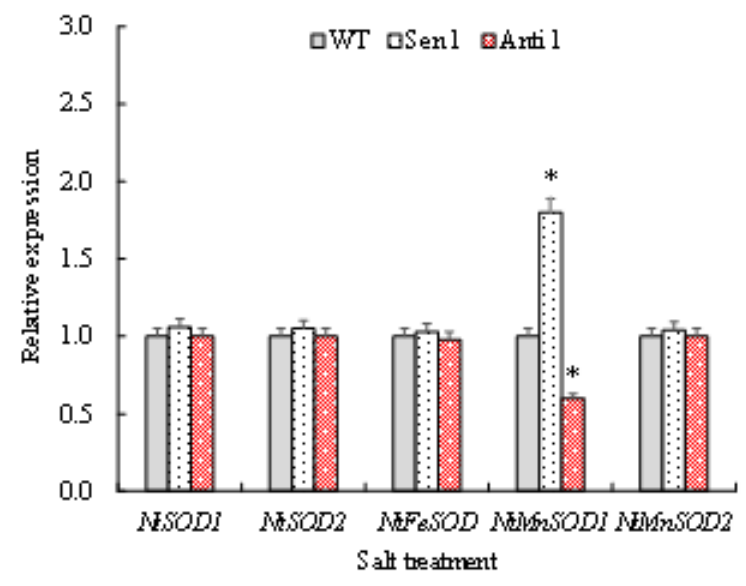

d

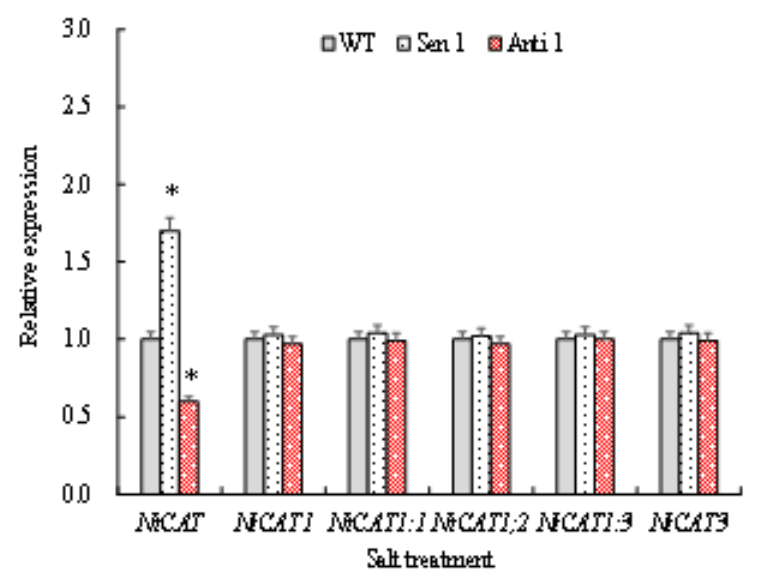

f

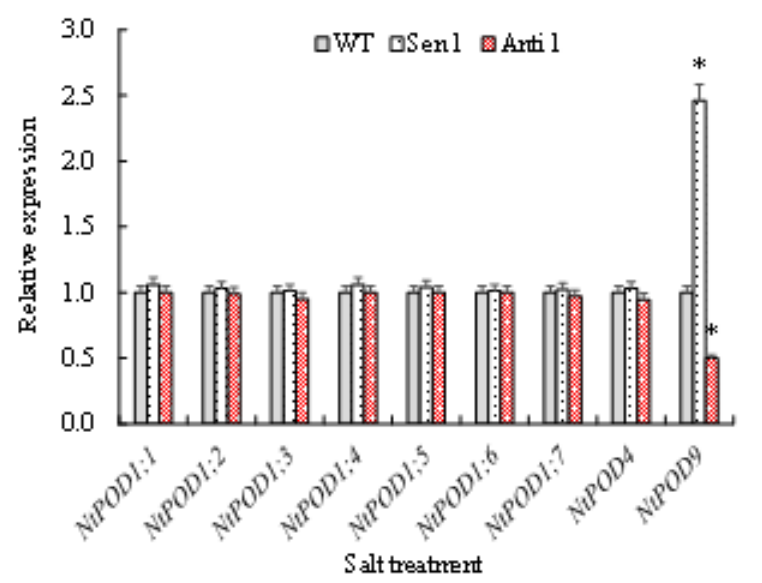

Figure 7 
Expression patterns of the genes in antioxidant enzyme families in the transgenic lines under drought and salt treatments

a, transcripts of SOD family genes under drought condition; $\mathbf{b}$, transcripts of SOD family genes under salt condition; $\mathbf{c}$, transcripts of CAT family genes under drought condition; $\mathbf{d}$, transcripts of CAT family genes under salt condition; $\mathbf{e}$, transcripts of POD family genes under drought condition; $\mathbf{f}$, transcripts of POD family genes under salt condition. Data shown are average plus standard error with symbol * to represent statistically significant compared with WT $(P<0.05)$

a
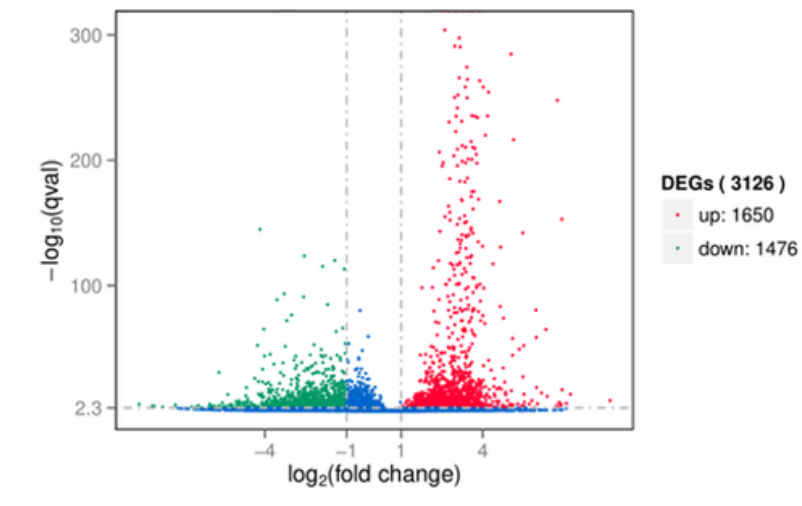

c

down: 1476

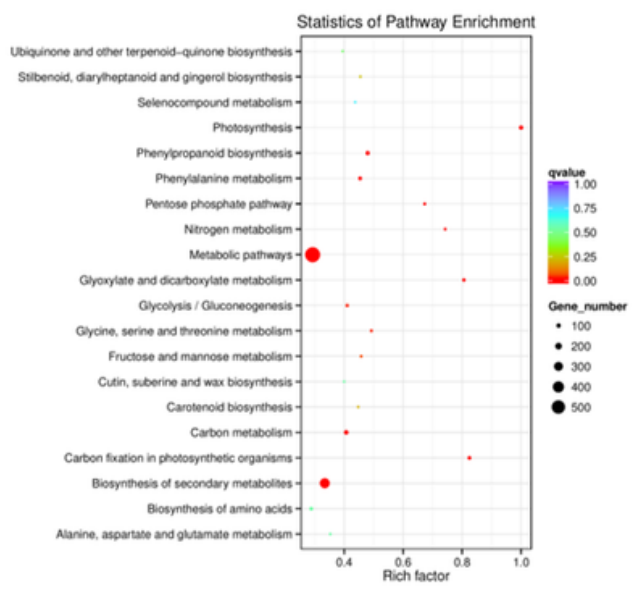

b

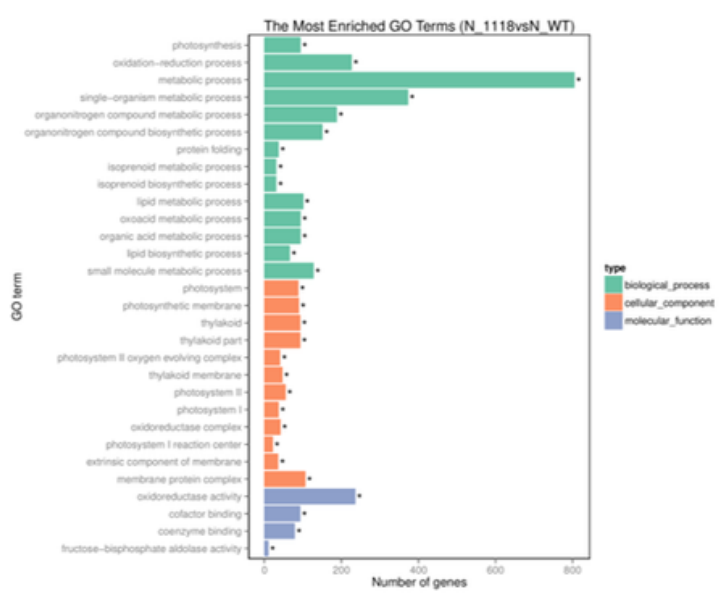

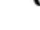

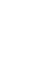




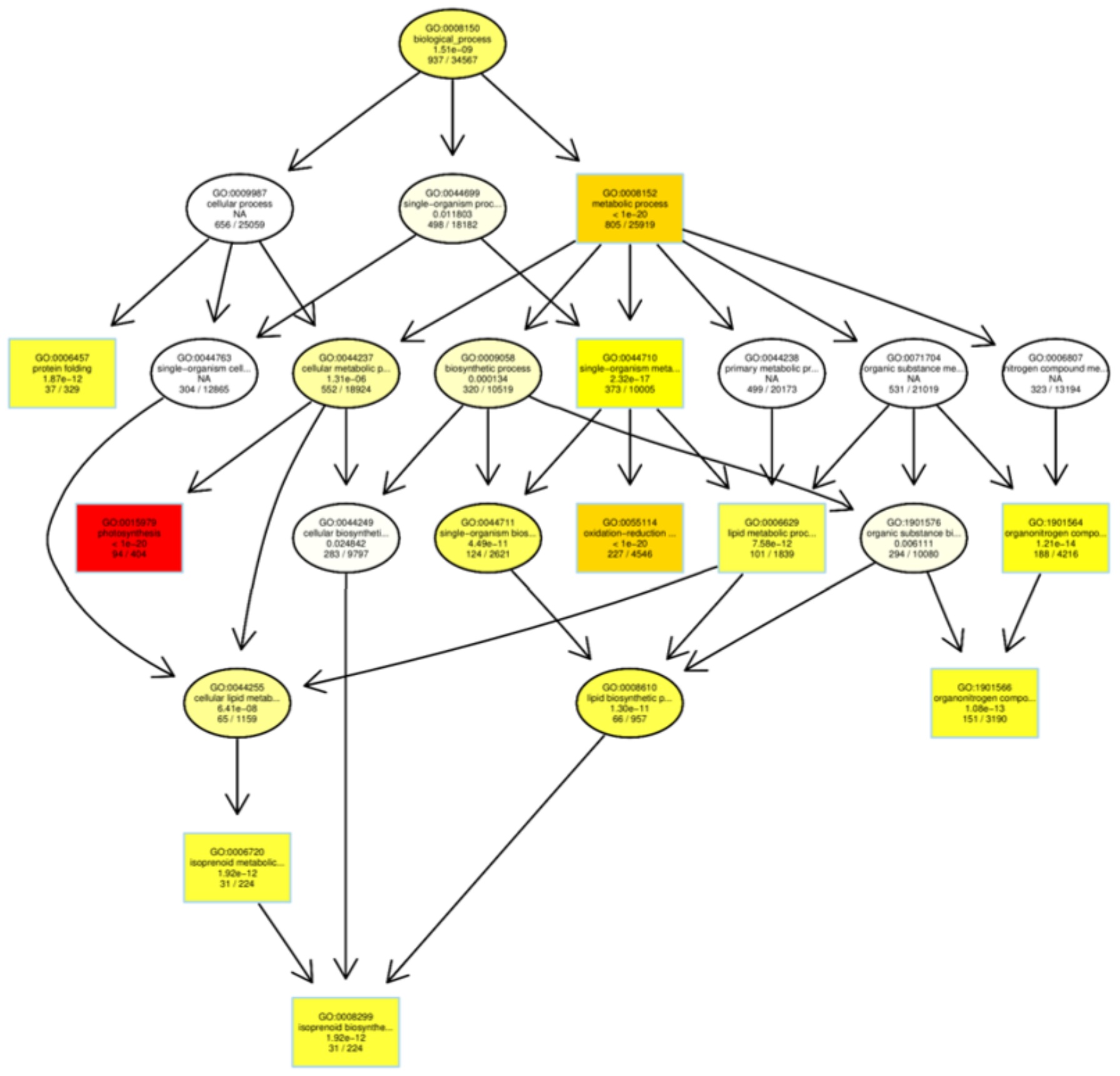

Figure 9

Putative diagram for biological pathways associated with plant drought response underlying modulation of TaMIR5062

\section{Supplementary Files}

This is a list of supplementary files associated with this preprint. Click to download.

- Supplementaldata.docx 